\title{
Declarative Consciousness for Reconstruction
}

Leslie G Seymour

PersInVitro, LLC

San Jose, CA, USA
Seymour@Persinvitro.COM

Editors: Randal Koene, Diana Deca

\begin{abstract}
Existing information technology tools are harnessed and integrated to provide digital specification of human consciousness of individual persons. An incremental compilation technology is proposed as a transformation of LifeLog derived persona specifications into a Canonical representation of the neocortex architecture of the human brain. The primary purpose is to gain an understanding of the semantical allocation of the neocortex capacity. Novel neocortex content allocation simulators with browsers are proposed to experiment with various approaches of relieving the brain from overload conditions. An IT model of the neocortex is maintained, which is then updated each time new stimuli are received from the LifeLog data stream; new information is gained from brain signal measurements; and new functional dependencies are discovered between live persona consumed/produced signals.
\end{abstract}

Keywords: consciousness, LifeLog, mind extension, brain preservation, brain machine interface

\section{Introduction}

Within the last 10-15 years, research in two different disciplines paid special attention to the challenge of computationally formalizing and capturing the information content in the human brain. Computational neuroscience, launched the "whole brain emulation" (WBE) effort (Sandberg and Bostron, 2008; Koene 2013s and 2013b) while computer science launched a number of LifeLog R\&D programs (Bell G., 2004) (IPTO, 2003). While the WBE approach focused on attempts to accurately model neurons and neural systems by exploring their internal neural structures, LifeLog collected and computationally interpreted digital logs of sensory input and response data to / from the human body. Both of them point to a formal definition of the human mind and consciousness. The objective of the analysis in this paper is to discover ways of potential fusion of these two approaches for the benefit of increased accuracy, completeness and efficiency of deriving such formal definition. Completeness and accuracy of WBE imply that all structural neural components with any impact on who we are have been accounted for. For LifeLog, the internal biological structural coverage is not a requirement, but all details of observable human behavior are. A post fusion completeness would encompass both sets of requirements. But even if such superset of completeness is not a priority, the overlapping consciousness detail findings can be of tremendous help in speeding up the combined mapping process and in validating the partial results. Since LifeLog should be built with industry proven and matured information technology methods of defining layered abstractions of semantical 
definitions, an approach of utilizing this makes it easier to describe intermediate findings while the underlying neural structural mapping could still be in the works.

LifeLog R\&D commenced over a decade ago, pioneered by Gordon Bell's 'MyLifeBits' program at Microsoft Research (Bell G., 2004) and then, the social media explosion and Quantified Self (QS) community growth made it ubiquitous. With a few years of delay, the idea of using LifeLog data for analytics derived universal persona model data emerged (Seymour L. G., 2005), while monetizing fragments of such persona models became commonplace in the industry in the form of "personality profiling", "behavioral analytics" and "sentiment mining" for CRM ( Customer Relationship Management ), safety and security surveillance, and most recently for "personalized medicine" and life-style coaching. While tremendous application opportunities were discovered by utilizing IT models in psychoanalytic research (Oren T.I., 2003) the application of computing technologies in other human-centric disciplines brought fundamental transitions in these traditionally distinct fields, resulting in the development of computational biomedicine, computational neuroscience, computational genomics, computational 'Omics' ( Snyder ), etc. The convergence of IT persona models across traditional IT such as the original LifeLog efforts with the various adjacent, computational science domains is becoming increasingly a realistic opportunity.

From the biological point of view, the neocortex is typically viewed as the dominant biological constituent carrying the information that defines a persona model, in other words consciousness. A plethora of philosophical, ideological, ethical, legal work have been addressing the exact meaning of consciousness, and challenging the equation of it to something that can be modeled by pure and existing IT means. Nevertheless, IT and information science have proven to be the most effective disciplines to make tangible progress in this area, hence the popularity growth of the various, adjacent computational science disciplines. In order to gain a sound understanding of how the biological substrate of the neocortex is utilized in capturing the human personhood and identity, building an IT model derived from purely IT experiential data and attempting to map such model to the computational neuroscience model of the neocortex seems a promising effort.

\section{Assumptions}

\subsection{Why Declarative?}

The term "declarative" is used here to emphasize that as opposed to the "How", and to the underlying structure, the issue is a 'platform-transparent' definition and how to formulate it such that it could be conveniently mapped into a variety of substrate architecture

There has been an on-going discussion on the most appropriate discipline to use for establishing a theory of consciousness discovery research, including neural theory (Koene, R. 2012 and Gallant, J.; et.al;. 2013), electromagnetic theory (Berger, T.W.; et. al. 1995; Berger, T. 2013; Dunin-Barkowski, W. 2013); and quantum theory (Hameroff, S. 2013).

The first two of these approaches did demonstrate some limited success in correlating measurements and their analytical results with human observations of consciousness constituents. These consciousness constituents included personality traits (e.g. tendency of experiencing pleasure from observations of suffering by others), affective states (such as arousal, stress), and the ability to relate to certain concepts of known semantics (Gallant, J.; et.al;. 2013), etc. The 
fundamental success of a digital consciousness preservation technology depends on how independent the consciousness specification method is from the underlying consciousness preservation implementation structure and at the same time, how close such specification method is to the "ordinary" human perception of the individual's consciousness.

The term "ordinary" here in itself is subject to interpretation. A consciousness component is "ordinary" here, if the data representation of it is directly measurable using today's technology, or there is a known technology to derive from such measurements. If such derivation requires a sort of "reverse engineering" that is envisioned today but not yet available in commerce or in advanced technology centers, we don't consider it as ordinary. Figure 1 shows the layers of "ordinary" consciousness data components. Note that measurements at neural cellular levels or deeper, for example at the quantum level are not included, since we do not have the capability of satisfactory resolution and scope of measurements at that levels such that we could derive from them any of the "ordinary" components. DNA is however "ordinary" data, since sequencing technology is available and deriving "personality traits" from DNA data is also possible today, while such personality traits have been known in the field of psychology and many of them are used in common sense interpretation of personal behavior.

\subsection{Experiential data vs. Consciousness}

Similarly frequent discussions are taking place in social media, for example in FaceBook groups such as (Carboncopies), arguing that experiential data, such as images seen, speech, sounds heard, letters read, etc. are not representations of THE consciousness. Here it is understood that while such data indeed does not constitute consciousness in itself, however its semantics in the context of the life of the subject, and in the context of its relations to other experiential data and to higher level concepts derived from such experiential data do constitute consciousness specification data.

\section{High Level Objectives}

A longer-term, transhumanist objective is to provide complementary solutions to other brain preservation methods based on computational neuroscience and/or biomedicine. Examples of the latter are cryonics and chemical brain preservation. Digital consciousness data is often anticipated to play important roles in brain repair and reanimation validation efforts in support of these alternative brain preservation methods.

Regardless to what extent transhumanist objectives are pursued if at all, the completeness and fidelity level of digitally captured information on persona models, identity and consciousness are foundational research objectives supporting research efforts in a number of other related areas. A relatively shorter term and "mid-tem" objective of such efforts is to facilitate more measurable progress in several areas of longer-term goals, including

- brain mapping, where the purpose of the consciousness reconstruction is understanding the brain;

- mind uploading, for revival to mitigate pathological conditions, including clinical death

Shorter term electronic consumer and business product opportunities can also benefit from digital consciousness capturing for applications in personal productivity and security 
improvements, where the scope completeness and fidelity level of capturing the consciousness could be important value proposition factors in the market success of these new products.

These objectives are broken down into a number of more specific objectives and described in the Section "Detailed Objectives" below.

\section{Existing Research Efforts}

A number of existing research and development efforts are based on specific models of the neocortex and the broader brain in general, although with no focused intent to map digitally captured personal experiential data to these:

- Numenta HTM: Hierarchical Temporal Memory (Hawkins, J; et.al. 2011)

- Open-source AGI software models of consciousness such as the purely AI functional model "OpenCog" and its more recent versions that also consider some of the computational neuroscience based structural representation of the functional model (Goertzel, B.; et. al. 2013).

- "Recognizable Cognitive Behavior" by SPUN using the SPA, Semantic Pointer Architecture (Eliasmith, C 2013)

- Kurzweil's PRTM (Pattern Recognition Theory of Mind) architecture described in (Kurzweil, 2012). PRTM is derived from the more generic model of HMM and abstracted from the results published by the most commonly known computational neuroscience research of how the neocortex works.

\section{High Level Architecture}

In this section the persona model as an information technology approach of consciousness is explained, first in context of other disciplines, then by presenting its constituents and finally showing how it is proposed to be maintained in several architecture layers.

\subsection{Why is Consciousness an IT Persona Model?}

The definition of consciousness is often a function of the investigator's primary discipline of work. Computational neuroscientists would prefer to view it as a WBE model at the neural level or close to it, IT workers view it as an IT model of a person, EMF models and Quantum models are also often promoted based on establishing a structure of physical signal measurement results.

Numerous previous published works inspired the author to view consciousness as an IT model.

1. John Von Neumann's 1958 landmark work, The Computer and the Brain ( Von Neumann, J, 2000) explicitly made comparison between the biological brain and the digital computer architecture. The takeaway here was the emphasis on the irreconcilable differences between the mind and the computer as viewed by von Neumann at that time. Nevertheless, von Neumann initiated the discussion on the subject, thus at least, acknowledged the validity of the question. 
2. Founders of information theory defined entropy as a quantity of new information resulting from a system consuming some known information and then producing another known quantity of information that was unknown prior to receiving and processing the input information. Here the "system" was viewed as any kind of system, including living systems, individuals and/or communities of them.

3. Gordon Bell made the infamous "I Am the Data" statement in his keynote speech of the ACM MM at Columbia (Bell, G. J. 2004 )

4. Martine Rothblatt of the Terasem Foundation is often speaking of "Mindfiles", as the digitized log of complete brain information content.

5. Natasha Vita More of Humanity Plus has been speaking about primary or secondary consciousness, as people's experiential perception of a person. This paper argues that a person's perception can be captured in digital LifeLog data with subsequent personality analytics processing.

6. An Australian speaker at Singularity Summit 2009 in NYC, used the term of Reconstructive Consciousness indicating personhood preservation based on multimedia LifeLog data.

7. Max More, a leading transhumanist and consciousness preservation scientist was speaking about LifeLog based digital personhood data preservation to supplement bio-medical brain preservation approaches. ( More, M. 2013 )

8. Kurzweil in his 2012 book "How to Build a Mind?" ( Kurzweil, R, 2012 ) described The Pattern Recognition Theory of Mind (PRTM) that can be modeled by a new computer architecture the "Principle of Operations" of which was described in his book. The author here views Kurzweil's work as a redo of von Neumann's book half a Century later, when we already know enough about the HMM-based pattern recognition architecture both in the brain and in decades of successful in-silico applications for recognizing enough equivalence between the two substrates to state that it should be feasible to integrate the two. The purpose of the integration here is envisioned for the purpose of brain extension ( Kurzweil, R 2012), or for transitioning the mind to an alternative substrate ( Koene, R 2012 ), and/or to supplement the currently already practiced bio-medical brain preservation efforts (de Wolf, C 2013 and More, M 2013 ) as also illustrated here in Figure 7.

9. New consumer product marketing approaches have recently advocated the (initially informal) definition of "persona" (types) as use cases of new product market requirements targets. After the market requirements definitions describe who these persona types are, the engineering product requirement definitions determine, if these persona types will be served by: providing personalization options of the new products; new product versions to be released for different persona types; or by differentiating by different service packaging.

Many of these sources used the term "consciousness" as a real life entity that does have a fully equivalent information model, including the speaker at the Singularity Summit '09, Max More, Natasha Vita More and Martine Rothblatt.

The author himself has been exposed to these thoughts since the late ' 60 s as they became accessible. In 2000, the author wrote a "Personality Engineering" research program proposal to model a person's full behavior on a computer. The author presented the idea of LifeLog based "personality" model harvesting, first at the 2005 ACM Multimedia Conference with Gordon Bell's "I Am the Data" keynote speech ( here in Figure 3 ), then in more details at the $2^{\text {nd }}$ CARPE Workshop ( Seymour, L.G 2006 ), and also started the "Reconstructive Consciousness" 
Facebook group in 2009, after picking up this new term at the Singularity Summit earlier that year.

\subsubsection{IS THE SubCONSCIOUS THE ANTIDOTE OF CONSCIOUSNESS?}

These two concepts seem as opposite based on the common sense usage of English. But this also seems a little confusing question first, because it would seem obvious that we can subconsciously act but still process in ways that are typical to our personality. Many of the consciousness research disciplines are typically making use of former, common sense interpretation. For example, during his Socrates interview in the Singularity Hub, Hameroff ( 2013 ) argues that when he is driving on a low-traffic expressway subconsciously, so to speak in autopilot mode, he is unlikely to use his reasoning intelligence of his neocortex but still, random sophisticated thoughts are going through his mind. Consequently, he argues, consciousness must be something beyond the neural structure of the brain, must be something that could only be explained at the quantum levels. EMF consciousness theories using electro-magnetic signal measurements to differentiate whether the subject is under anesthesia or he / she is conscious, thus providing a good use case for investigating the nature of consciousness.

Since AGI is considered part of computer science, the author here is taking the liberty of using set theory, state machines, OOA/D and soft computing models to approach the relations between consciousness, full self-awareness, subconscious, sleeping, dreaming, unconscious, under anesthesia, coma and biomedically "suspended" - the latter being a term used in cryonics after clinical death is diagnosed.

In the simplest paradigm, consciousness is what the person is and the rest of the terms in the list above signify different states of this consciousness. The aggregate of the behavior of the person in all of these states is what constitutes the person's overall consciousness.

The next important step in refining the model is adding the needed realistic ambiguity to these states. Clearly, the person is or at least could be in more than one states at a time, e.g., waking up gradually, etc. Most common models here would include Bayesian networks adding probability to each state, but other options could also be useful to apply, such as fuzzy states and fuzzy state transitions. Patten recognizers assigned to different states could drive these transitions

The use of inheritance relations for defining the different consciousness states could provide the most generic consciousness model definition at the highest abstraction level.

Most importantly, in high fidelity level models, ambiguities are assigned not only to entire consciousness states, but individually, to each piece of concepts which may include both data and function. For example, during the expressway drive, some of the roadside visible objects may represent objects of some degree of awareness. These are the factors represented by weights and inhibitors connected to the pattern recognizers of the neocortex. We are fully aware of some of the objects, but barely of others. We see the car in front of us, but the roadside bushes, trees and houses are also in view.

\subsection{What is a Persona Model?}

A "Persona Model" is a formal software specification of a complex object representing a specific human person. A generic abstraction of this is the persona model applicable to a certain group of persons, or to all persons. Further to this, more generic abstractions could also include living species other than humans, although this would point beyond the scope of the analysis in this paper. 
The specification techniques and standards here are the same as the ones successfully used in the software industry including RDF as applied in Web-semantics, UML with all data and state machine models, data and knowledge base models, predicate logic statements and function point definitions, soft computing models including stochastic models, fuzzy, neural network and genetic algorithmic models and biological colony interaction models.

The description of such "Persona Model includes

1) Experiential hypermedia content that the individual was exposed to or he/she was producing. This includes

a) data that has been retained in the brain as memory, although the state of such storage of this data in the memory may not be readily available in the process of building the persona model.

b) Data produced by the human body, such as medical and/or "quantified self" (QS) measurements. This would also include data derived by means of genomics, bacteriomics and other "Omics" ( Snyder M, 2012 and Snyder M. et.al. 2012 )

c) Environmental data to which the human body is exposed to;

2) Contextual metadata linked to the above data components, such as location, time, real-life events in the surrounding environment, known related concepts / memes etc.

3) Psychological behavioral model data: personality traits and states, affective states and facets, incentives, objectives and interests, IQ, emotional intelligence, cognitive and intuitive performance (Seymour, L.G 2005 ). These can be derived from the above data using Big Data analytical methods, including Machine Learning (ML).

A complete persona model in this sense has not been recognized in biosciences, to the author's knowledge. Separate components of such persona model are however important factors in rapidly advancing areas of biosciences. Typical examples are here

a) Medical laboratory measurements

b) Quantified self measurement data

c) “Omics” research and therapies ( Snyder M, 2012 and Snyder M. et.al. 2012 )

d) Brain fMRI to map out

a. concepts in the neocortex,

b. psychological attributes ( e.g. arousal in music, experiences pleasure of seeing others suffering, etc. )

e) EMF measurements to classify states of consciousness

Some of this research is integrating many of these components with one another and sometimes, also with contextual data outside of the bioscience domain. These are however mostly narrowly focused correlation research and no known integration exists just for the sake of arriving at a comprehensive, single model of a specific person.

\subsection{Layers of Representation of the Persona Model}

Figure 1 illustrates the different category layers of data as constituents of a human person's mind and behavior. In more details, these were described in some of the previous investigations on 
how to represent a person as data (Seymour L. G., 2005), The top layer, “Observed Experience Content Representation" gained as direct personal observations by various Lifelogging devices, such as the currently emerging Google Glass and Memoto wearable devices and technologies (Bell G., 2004) (Seymour L. G., 2005), and in a broader sense, using Quantified Self ( QS ) data input. These do not require any special professional domain expertise to interpret when consuming the data by human sensory organs, although in some cases, some QS measurement interpretations do assume some degree of understanding of some fields of medicine. The second layer, "Knowledge Objects / Semantics" do require the use of AI analytics techniques and tools to derive computationally from the top layer data, but again, it does not assume any specific target domain expertise, except the ones requiring the interpretation of the QS measurements. The third layer can also be obtained using analytics running on the data of the adjacent data layers, mostly assuming the understanding of notions defined in the field of psychology. The last layer (Snyder 2012) is sourced by biomedical methods and they often may correlate with data in the psychology domain.

\begin{tabular}{|c|c|}
\hline \multicolumn{2}{|c|}{ Observed Experience Content Representation } \\
\hline \multicolumn{2}{|c|}{ Knowledge Objects $/$ Semantics } \\
\hline $\begin{array}{c}\text { Objectives, Incentives, } \\
\text { Interests, Reactions, Focus } \\
\text { Intentions and Motivations }\end{array}$ & $\begin{array}{c}\text { Cognitive / Affective Traits, } \\
\text { Facets and States }\end{array}$ \\
\hline \multicolumn{2}{|c|}{ "Omics" with Genomics (DNA and Epigenomics) in them } \\
\hline
\end{tabular}

Figure 1 Consciousness Definition Data Layers

In order to transform raw digital measurement data into a format compatible with how we believe declarative consciousness specification data may exist in vivo, we need to distinguish four data representation stages in the architecture of this transformation, as shown in Figure 2.

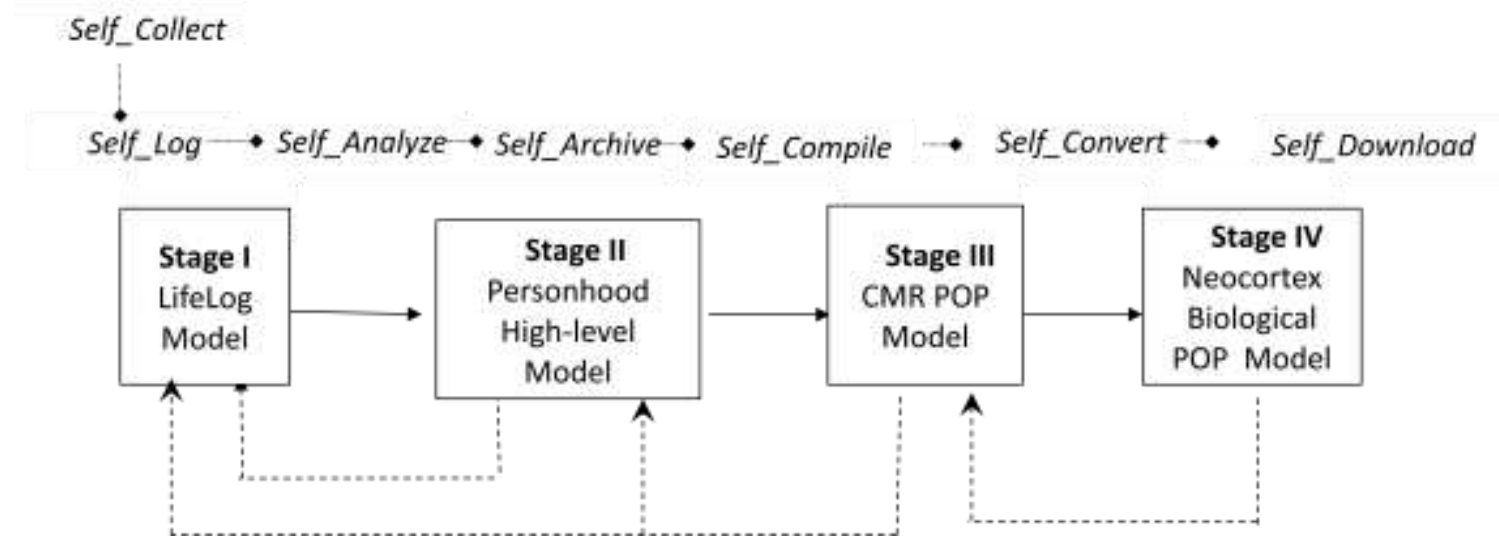

Figure 2. Stages of Consciousness Data Collection and Processing to Derive Consciousness Specification 
I. LifeLog: minimally processed experiential content suitable for direct human consumption (Bell G., 2004). This is the top layer in Figure 1. From the data processing flow point of view, the "Omics" layer also belongs here, even though biomedical domain specific expertise is required for its analytics.

II. Personhood high-level model derived from LifeLogs (Seymour L. G., 2005). This layer is derived by analytics from the first stage data and these were represented in the middle two layers of Figure 1. This stage has a feedback to the Stage II LifeLog level model for refocusing the raw data collection and $\log$, as more insight is gained on the completeness of the higher-level model, e.g. what type of data is missing. Similarly, since the models in the last two stages have more accurate information on what data is actually stored in the neocortex, they can provide focus to Stage I on what type information need more details. Stage II itself gets feedback from the later representation stages indicating the relative priorities between the higher-level model constituents.

III. CMR - a Canonical Mind Representation form. The term "Canonical" indicates here a lowest level common abstraction of the Principles of Operations (POP) of "machine languages" of the brain's neocortex (Stage IV below). The term also refers to the early years of high-level computer architecture research back in the 1970's, where a Canonical Interpretation Format (CIF) was proposed as the most efficient high-level language support machine language abstraction for a broad variety of micromachine architecture to implement on. The task here is a little more challenging though, since the definition of the personhood-level data model is today far from the degree of maturity of the already deployed programming languages forty years ago. Similarly mature were the instruction set architecture for computers and firmware development. Still, the optimality goal in this stage of research is highly important here, for a number of reasons. The first is the simplicity of mappings between known semantics and the neocortex level primitives. The second is the ability to assess the coverage and progress in capturing the mind of a specific person. Third, the maximum simplification of the mappings would also save computational resources for simulation / emulation, which is often viewed as a critical factor in progress of computational neuroscience research.

Stage II receives feedback from Stage IV on what actually has been embedded in the biological substrate of neocortex in order to update the CMR-level model and to inform the higher level and earlier stages to update their contextual priorities and focus

IV. Neocortex principles of operation (POP) model with direct map to the biological substrate. As listed in the section "Existing Research Efforts", there were a number of proposals and their implementations on modeling the lowest level building blocks of the neocortex, that the AGI developers would still fully understand for the purpose of mapping higher level knowledge with known semantics. The selection among these models and a potential fusion of them would determine the representation at this stage.

The need for Stages III and IV are further reviewed and justified in Section 5.5Why Not Compile LifeLog Level Data Directly to the Neuron Level Structures? For initial design analysis here, the abstractions of (Kurzweil, 2012) are chosen as the model in Stage IV) for several reasons:

- For the ease of mapping, this is the closest to the most recent understanding of the neocortex architecture, which has also been published with broad exposure and reviews. The PRTM POP can also serve as a directly mappable CMR to other, lower-level neocortex model building blocks, such HTM. 
- The PRTM building blocks functionally encompass the features used in a broad range of paradigms used in constructing intelligent systems following the assumptions of how the brain worked. The building blocks are modules of sets of neurons, with

- approximately a hundred neurons in them,

○ each module providing a template for recognizing a pattern

- a number of redundant modules assigned for supporting a concept,

- each module has input and output synapses, exciters and inhibitors as input to these modules,

- Inter-module connections are dynamically created and destroyed, based on the external stimuli driven results of pattern matching

Kurzweil and a number of other publications (e.g. Berger, T. 2013; Dunin-Barkowski, W. 2013) mention that the architecture of these neocortex building blocks are not identical, and they can further be subdivided into several classes of module types. In addition, there are several other important sections of the brain carrying consciousness specific data that are not directly represented in this PRTM model described in (Kurzweil, 2012). These were often referred to as the "old brain" the presence of which in the brain is not unique to mammals such as humans. But even animals without a neocortex (such as many types of birds) can exhibit intelligent behaviors to some degree, and that structures from an earlier period in our biological evolutionary history may well have been repurposed to take on very important roles. The hippocampus is in such an older structure, but is absolutely essential to our ability to form episodic memories, which are a crucial part of conscious self-perception.

Nevertheless, in this work, just as the author did in (Kurzweil, 2012), a simplified view is assumed, where each PRTM module behaves in the same way. Kurzweil attributed the highest level and most complex concepts to the human neocortex that developed in latest stages in the evolution and not managed by other species. Examples included communicating abstract concepts in written language. It is a reasonable research strategy to start with the neocortex only, with the expectations of the underlying implementation model also covering the simplier use cases, even though those functions could be more critical to the survival of the human person. Other types of PRTM modules within the neocortex and building blocks in other brain sections should later be added to the model described here, as well. In the mean-time, potential disruptive impact of these later additions on the method applied here needs to be carefully monitored.

The final neocortex PRTM POP image representation shown as Stage IV in Figure 2 will be stored and functionally executed in different substrate configurations as the technology development described here is progressing from its initial phase to the final phase envisioned in (Kurzweil, 2012). Going from the most complete phase to the simplest phase, these phases are:

A. The Mind Extension Deployment (MED) phase, where the PRTM POP machine implementation is fully configured as the reference description in (Kurzweil, 2012). Applying the current state-of-the-art of distributed computer architecture, MED is implemented with a seamless transition between the biological PRTM modules and the Cloud-based PRTM modules. Functions performed by the biological neocortex PRTM modules can be "delegated" to PRTM modules in the Cloud without any noticeable disruption in the functions of the brain. Similarly, new Cloud-based PRTM modules can also be added to the extended brain seamlessly. This configuration is also illustrated in Figure 6. 
Each person will have two physically separate agents deployed. The first one is Kurzweil's brain implant, a BMI (Brain Machine Interface ) device connecting the neocortex of the biological substrate of the brain to the 'in silico' Cloud neocortex.

The second agent is in the Cloud infrastructure and it may or may not be a physically distinct device assigned to the served human person only. The real-time access constraints here could be much more stringent and critical than in general for Cloudbased IT solutions. Traditional wireless voice and data communications infrastructure architecture is a good analogy here even though the operational requirements for brain extension could event be more mission critical depending on the type of functions shared seamlessly across the BMI and the Cloud. An analogous infrastructure architecture would include equivalents to VLR ( Visiting Location Register ) and HLR ( Host Location Register ) functional entities of the GSM wireless infrastructure networks (Balston, D.M. 1993) today, where subscribers were registered and their status was tracked and all of the information required to serve their needs were stored in these entities along with more provisioning specific network equipment, such as subscriber management and AAA functions, such as authorization, authentication and access control. Even in today's wireless communication infrastructure architecture, these functional network elements are increasingly decentralized and distributed in an underlying location-independent network layer, and they became part of Cloud-stored content. In many cases, it will be a subject to interpretation, if a person specific Cloud content management agent is an easily identifiable entity in the Cloud, even though it definitely exists, at least virtually. For example, pieces of content shared by a group of humans, or by all humans could only be pointed to from person-specific content. In other cases, shared content can be copied and personalized if needed. The significance of ethical and a number other social aspects associated with the selections for this types of partitioning are enormous and could be disruptive.

The functional allocation between the brain implant and Cloud-based persona agents and the communication protocol in between them will be the result of a complex trade-off resolution process, with the most important factors including privacy, implant size, weight and power consumption, security, throughput and response time as neocortexstored knowledge and other identity content is exchanged between the biological neocortex and its Cloud-based "in-silico" extension. For security requirements and design, the threat-analysis scenario portfolio needs to include cases when the Cloud or fragments of it are or are not trusted, and/or the same may apply to the embedded implant segment, or to both of these segments. Even though these factors would have the utmost importance in deploying this system, further details of them here would clearly point beyond the issue of human consciousness definition addressed in this paper.

Since any communication from the BMI might change the brain due to its existing plasticity in adjusting to new external stimuli, known IT methods of extending existing IT systems may result in unanticipated overall results here. In order to explore and mitigate this aspect, comparative benchmark studies might have to be conducted with control groups who

a) are not receiving new input;

b) are not aware of receiving new input but they are

c) are receiving new input and aware of it? 
It needs to be determined how does the experiential behavior of these groups differs from one another? Is it radically different from cases when the brain is currently adjusting to disruptive changes in the environment and in the use of disruptive technology products?

The results could also indicate a need for careful and only gradual introduction of new functional extensions of the neocortex. The logistical process details of introducing new types of extensions here might also be similar to the current FDA approval process.

B. In this phase, there is a BMI implant, but no extension in the infrastructure, such as the Cloud. The implant acts as a gateway between the biological neocortex and the PRTM POP simulator described in the initial phase below. There is no actual neocortex backup or extension taking place, the CMR simulator in Figure 6 can however perform measurements, generate logs and diagrams indispensible for developing later the inCloud portion of the extended neocortex.

C. In this initial phase, there is no BMI at all, no implant, no wearable's and not even periodical fMRI sessions and analysis. The PRTM POP CMR machine has a bare-bone rudimentary diagnostic simulator, equipped with a PRTM-based knowledge browser with dashboard GUI and interactive, concept semantics explorer. For research and related product design and evaluation purposes, the user of this PRTM POP simulator can visualize the data need to understand how much of the 300 million PRTM modules of the neocortex is allocated for what semantical purposes, how much are unused, if any, and what were the access statistics per modules, the dynamics of purging any least recently used content. More advanced diagnostics features, could also evaluate, how "much pressure" is building up to "outsource" neocortex content to an external backup such a the Cloud, otherwise valuable affective states, goals, focus, etc. are likely to be compromised.

In this consciousness data processing flow architecture, an incremental mapping of the personhood model is intended into the CMR form of the neocortex model as also illustrated by the feedback data flow lines in Figure 2.

\subsection{Why is IT-based Persona Model Needed vis-a-vis Using Other Disciplines}

As earlier noted, the definition of consciousness is often a function of the investigator's primary discipline of work. Computational neuroscientists would prefer to view it as a WBE model at the neural level or close to it, IT workers view it as an IT model of a person, EMF models and Quantum models are also often promoted based on establishing a structure of physical signal measurement results.

The IT based Persona Model is a "Black Box" model of the consciousness, while the other disciplines based approaches are providing "White Box" models. The black box model is the specification of the object by describing its behavior without the need of understanding the internal structure of the implementation. The white box models capture the internal structure of the implementation. A validated, high-fidelity level persona model can not be built without using "Black Box" models. This has been proven by the software industry in the last fifty years, whenever a relatively complex system had to be built and maintained.

Pushing progress using only white-box models has always been tempting in system development. In the early years of the software industry, the best approach was for talented programmers to understand the problem and then directly cutting the implementation code that 


\section{DECLARATIVE CONSCIOUSNESS FOR RECONSTRUCTION}

worked at the first execution. As applications were getting more complex, with longer life-span, and exposed to more and more people of various background and requirements changing in time, this technique was not sustainable any longer.

The initial software technology maintenance approaches were similar to the current regenerative medicine views, which is a very promising approach for the cure of many diseases and prolonging life by focusing only on certain organs at a time, or by applying generic cure at the cellular level. Similarly, in the maintenance of complex legacy software systems, their lifespan could be extended by looking for special conditions in the code sequences and replacing them to match new application condition requirements. A well known example of such "cure" was the enormous efforts of "Y2K" program, before the new millennium started. These could have got accomplished without fully understanding how the entire system worked, not even at the top level of the black box system specification. But after too many patches and with radical changes in the overall system need, such approaches were unsustainable. Black box definition driven top-level, modern specification method compliant architectural constructs had to be built, and then, saving and using as much legacy code as possible as reusable plug-ins.

Building and reverse engineering the brain is the most challenging system engineering undertaking in history, when considering the complexity, the unknowns and the risks. A reliable approach to understand and/or build consciousness should combine both black box and while box approaches. This is illustrated by the author's view presented at the Neuro-Bio-Cogno Convergence 2008 unconference, as shown in Figure 7.

It is an important purpose of this paper to show in more details that iterations of balanced refinements of both in the black box model and in the white box model and mutual feedbacks in between them will result in the optimal pace of progress in modeling consciousness. Such combined approach has the best guarantee for minimizing the risks of branching out into deadend deep dives with irrelevant specifics. Such risks of wasting efforts are realistic both in the black-box and in the white-box approaches, if one of the two gets too much ahead of the other one in development progress, without using the other one for the much needed validation. These validation efforts can not only confirm the correctness of the other discipline's models, but it could also become an inspiration to explore new approaches in the refinement, refocusing and improvements in those models.

\subsubsection{Is Consciousness Preservation a Subset of Mind Preservation?}

The author views consciousness and mind as synonyms here. Appreciating the difference in the linguistic semantics, there is more ambiguity in the first one and the biological allocation balance is likely to be different between the two halves of the brain. The IT model toolkit use allocation between soft-computing and "hard computing" is also anticipated to be different. The mind is anticipated to have a more rigorous reasoning and focused objectives driven content while the "consciousness only" type of constituents seem more intuitive and ethical in nature. Since there is sufficient work now out there in areas such as sentiment mining, artificial intuition, emotion machines, and computational ethics, etc., the boundary line between these two types of "personhood" defining information is a gray area and it has no particular significance at this stage in building a digital model of the person. But, it could have more specific impact, if the interpretation of these terms are different in the requirements for the model.

If by "consciousness", we mean fully aware consciousness as a consciousness state as explained in section 5.1.1, it is possible to perform a partial preservation by duplicating a white box model on a different substrates and validate it only for the fully-aware state. A black box IT 
Persona Model can be extended to describe behavioral switches to other states and then specify certain state transitions as disabled, such as falling asleep, etc. As long as such transition switches are correctly mapped to the duplicated white-box models, the consciousness preservation is limited to the fully aware state only.

If the terminology is defined such that the mind is a sum of all knowledge and behavioral facets that the subject shares with other people plus his own consciousness including his individual "knowledge" and behavior which are different from any other members of his/her groups of people also modeled in the system, modeling only the individual delta of the mind could be possible and it could lead to significant savings in the storage space of the preserved mind for all people. It would also reduce the cost of updating the common part of the knowledge and behavior for a group of people.

\subsection{Why Not Compile LifeLog Level Data Directly to the Neuron Level Structures?}

When selecting a compilation target level model, the question is arising, why not use the brain's neural structure as is, the brain already has other levels at which one could choose to select for "identical" components reused throughout, e.g. synaptic junctions of a select number of different types with discrete pockets of neurotransmitter, neurons of several types with identifiable (though not actually identical) morphology, So why a level as abstract as the CMR? Well, there are a number of reasons, why an intermediate level of representation model, such as the CMR is needed, as opposed to compiling LifeLog level persona model data directly into a neuron level architecture:

i. There are a number of open issues, wrt how exactly the neuron level architecture works and our knowledge is evolving quite rapidly, as the pace of progress in neuroscience is increasing. We definitely do not want to change a complex compiler such as the MLB$>$ CMR compiler shown in Figure 6 each time we discover something new at the target level here. While it is conceivable that some new discoveries in the neural level will necessitate adjustments in the common CMR definition, hopefully "backward compatible" extensions only, certainly many of them will not mandate to make such changes. For the latter, only the interpretation of the CMR level code is changing, i.e. on the biological substrate, the bio-silico "adapter" will only change for the latter case. In the CMR simulator use, the simulator interpretation code is changing only for the first case, when the CMR level definition itself is changing.

ii. We may not even be sure, if the neural level architecture is common for all individual persons, or even for the same person as life goes by. If so, these could further aggravate the above constraint.

iii. While computational neuroscience is developing fast, along with its applications to assist the brain, so are the potentials of disruptive developments in genetic and cellular engineering. The results of the WBE for the brain that has evolved in millions of years may not apply as is for the times after the emergence of synthetic changes in the brain structures.

iv. The CMR level persona model data is more compact to store and transfer, than a fully expanded neuron level mapped data.

v. While for replicating minds, it is much safer to map neuron to neuron rather than going through an intermediate format, the results are questionable if we are not sure what the data actually represents at the neuron level. In other words, before the WBE program is 
reliably completed, it safer to deal with a level of model the semantics of which is well understood, since known engineering practices were used to define it. The architecture proposed in this paper for the $R \& D$ process to develop the solution as illustrated in Figure 6 serves exactly to arrive at a point, where "white-box" to "white-box" systems can reliably be copied across different substrates. In the mean-time, a combined "blackbox" \& "white box" solution is required, the need for which was described and explained in Section 5.4.

vi. The dilemma here is analogous to the early years of the computer industry, when unknowns and ambiguities existed both in the highest levels for programs and in the electronics architecture level. Some high-level programming languages existed, many more were in the works with no end of them visible yet. The portfolio of applications of various nature was also rapidly expanding. Firmware was invented to adjust the potentially unlimited varieties of computer hardware products to the existing supply of software. And yes, the machine instruction set, their Principles Of Operations became the stable control point, ensuring innovative progress both in new hardware development and in developing software for new types of applications eliminating the need for redevelopments of all solution layers each time the application need or the hardware requirements changed. Even though, direct compilation from high-level language code into microcode would have produced much more efficient code. Once a new hardware prototype was working reliably in the lab, volume manufacturing started and shipped with the latest version of the firmware assuring the compliance of the shipping hardware with the machine language specification. Changing the hardware to a modernized version and with an updated firmware was equivalent to copying the solutions to a new substrate. The machine language was equivalent to the CMR model. They were changing too, but only across competing vendors, radically new product families and/or classes of machines, controllers, minis, mainframes and supercomputers.

\section{Detailed Objectives}

The design of an on-going compilation of LifeLog experiential data into a CMR representation will be driven by a number of specific objectives, as described in this Section. The objective numbers here are also shown in Figure 6, assigned to the data, control and impact flows.

1) Validate LifeLog on completeness.

The content completeness validation requirements and tasks need to be specified and evaluated at least in two dimensions: along the different stages of representation of the consciousness data as shown in Figure 2 and along the different development phases of the PRTM POP machine configurations as described above in phases A, B, and C.

One of the most valuable completeness estimate is for the data representation Stage II shown in Figure 2. This is where a user can have an intuitive assessment if everything got captured in terms of concepts that are ubiquitously familiar in the context of an ordinary human life. In the initial development phase, the expectations will have serious limitations, since initially it will be hard to estimate, how much of this content got actually captured in the neocortex in stage IV. A content captured in the raw measurement data Stage I may or may not persist in stage IV, depending on a number of factors: was the subject aware of the data or not, was it in the focus of his interest, and finally, was the available neocortex PRTM module pool open enough for accepting the 
new data, or it was already saturated with higher priority data. The latter factor is more likely to loose its significance in the most complete, MED development stage A, since at least theoretically, anything and everything can be immediately uploaded in the extended, 'in-silico' neocortex extension.

This would bring up the question of why bother assessing the completeness in today's early stages of development, when we suffer at least from two significant limitations of technology progress. First, AI/AGI is developing fast in terms of recognizing concepts in multimedia, text and other modalities and relating personal experiential content to common, public domain content, but we know it is only a small fraction of what will be available in fifteen years from now. Second, although there is a promising progress in spotting concept maps in brain fMRI image analysis (Gallant, J. et. al. 2013), the actual coverage will be much easier to measure directly with brain implants in 10-15 years. However, there is a necessity of immediate completeness assessment, in that a significant portion of contextual information and semantics is still available and accessible right now at the time of the observations of the personal experiences are made, but much of it is likely to be lost by the time the AGI / BMI technology is significantly better developed, at least in the area of personal brain preservation of today's population.

2) Harness PRTM module pool allocation progress by using fMRI analytics

(Gallant, et. al. 2013) and other potentially available similar techniques in the future, that can be used to determine what content got actually embedded in the neocortex. Theoretically, if such fMRI analysis provided $100 \%$ complete and accurate coverage change results, it becomes possible to determine the entropy difference between the accumulation of information in stage II and stage III as shown in Figure 2. Stage III, the CMR stage with simulator would then be able to monitor and diagnose what the missing information is and devise what to do about it. This objective is a prerequisites of meeting the above completeness validation objective 1).

While trying to achieve this gap analysis goal by fMRI analytics, we need to be aware that many analysis techniques are quite intrusive. For example, fMRI analytics is not a process that goes unnoticed by the subject. In fact, it is likely to introduce episodes of extreme significance to that person's memory. The act of undergoing the analytics procedure becomes a salient cause of plasticity and modifies that person's experience not just of that episode in life, but also of any memory recall undertaken during the procedure.

Mitigating this side effect, comparative benchmark studies could be conducted, similar to the ones suggested for discovering brain plasticity compensation need while external BMI stimuli is injected, as described in Section 5.3, in Phase A of the Stage IV deployment for Figure 2). As significant improvements can be anticipated in the advancement of the visualization technologies, less intrusive, more seamless and passive imaging methods are also likely to develop to replace the current, episodic fMRI sessions introducing these side-effects.

\section{3) Analyze subsequent logs}

in Stage I and the resulting updates in the personhood model II in order to determine, update what got embedded in the neocortex Stage IV. This objective is a prerequisites of meeting the above completeness validation objective 1). This is especially important in 
the absence of concept capturing feedback by brain fMRI. In this case, AI tools for related conversational semantics analysis can be attempted to apply and indirectly to infer from the information communicated by the subject person, whether a previous piece of information was captured, processed as an anticipated concept, or not.

4) Diagnostic" conversational feedback for validation

The use of targeted, subsequent active "diagnostic" conversational feedback for validation of neocortex content is equivalent to the technique to achieve the previous objective, but it is not based on the analysis of passively generated communications logs. Instead, a diagnostic administrational component would pose a preferably perceived as passive user friendly challenge to the subject such that at least partially, the purpose of the challenge would be solicitation of responses that could clarify if a previous piece of concept got captured in the neocortex as previously anticipated. Further guidance in managing this objective is outlined in Section 8.2.1 How to minimize the active diagnostic communication overhead and in Section 8.2.2 Diagnostics Impact on the Personality Changes.

\section{5) Biomedical and LifeLog derived content fusion}

At the end of the functionally operational life cycle of the brain on the original biological substrate, an optimally "fine-tuned" CMR mind file should be available and stored for the purpose of fusing it with the results of activities in the previously suspended and then reanimated brain if and when such technology is available. Biomedical cryopreservation is the most commonly known such technology (More, 2013). The cryonics community is sometimes considering another possibility of repairing neural damages in the suspended brain, most likely using nanotechnology processes prior to reanimation. According to another, related scenario, the suspended brain could be "reverse engineered" to some degree using yet TBD scanning technologies, such that more and more detailed and complete neocortex - concept mapping is possible to rebuild as technology is improving during the suspension compared to the map available at the time of the suspension. In this scenario, the iterative compilations from the high-level personhood stage model II illustrated in Figure 2 to stages III and IV can continue while the patient is in suspension state. The improved stage IV model can either be immediately applied to the suspended brain in case such biometrically preserved neocortex neural update technology is available, or just continue the iterative improvements using simulation at all stages, thus iteratively accumulating the improvements through performing end-to-end simulation cycles with feedbacks illustrated in Figure 2 and Figure 6, In the latter case, a much better quality Stage IV model is available, when the BMI implant based MED support milestone is reached.

\section{6) Assistance by gateway implant based upload.}

As described in the MED scenario in (Kurzweil, 2012), the implant will continuously "delegate" portions of the newly emerging brain tasks to its Cloud-hosted extension, since on the biological substrate, the neocortex capacity is saturated at early age. Therefore, the iterative, end-to-end content maintenace illustrated in Figure 2 would contribute an additional bonus feature to BMI supported seamless "mind extensions": not only the neocortex capacity limitations are lifted, but additional content can also be captured, i.e. what content might be missing from the subject's external context, which was important but did not get captured in the bio-neocortex because the subject was 
unaware of it, had no interest, etc. Such "auxiliary" content would most likely go directly to the Cloud extension, although hard to say for sure. The end result is broadening not only the subject's attention span, but also the scope of the sensory input. For example, while attending a public event, auxiliary information from the Web can also be retrieved and integrated at Stage II, while the subject could continue his or her life with the auxiliary information in the real-time accessible portions of the brain in either forms of substrate.

It should be noted that not only might augmentation by auxiliary systems broaden effective attention span and scope of sensory input, it may also significantly alter the experiential life of the subject and therefore the subject's person. This is not necessarily an unwanted possibility, but it is a possibility which should be carefully monitored.

7) Repair of Alzheimer's damaged cell by reloading missing content,

when short-term gateway implant is available. This objective can be viewed as a special derivative case of the reanimated brain support requirement 5) above. Here the damage can occur gradually and continuously while the data collection and repair are also performed as the brain is functioning in more than one substrates.

(Berger, T.W. 1995) describes a VLSI hippocampus implant which functions as a gateway between short term memory and long term memory on the biological substrate of the neocortex. Today it is not clear, if Kurzweil's mind extension to the Cloud vision will be based on a BMI gateway as an implant that could be integrated with Berger's VLSI hippocampus, or not. If the two are integrated, or at least, they can communicate with one another either directly within the brain, or through an 'in silico' external infrastructure, there is a straight-forward way of addressing the Alzheimer's damage reconciliation problem. When loss of neural cells or their connections would prevent from retaining more recent signal represented experiences, such content can be immediately referred to the "extension gateway" implant for storing it operationally in the could extension. If it was a "high-priority" experience content that should be stored in the biological substrate, the "extension gateway" implant core functionality can take care of it, since it should have cache management between the two substrates for serving healthy brains, as well. time:

It is important to note two limitations here about Berger's neuroprosthesis at this

1. It has been tested in very carefully controlled experimental conditions, where it manages to accomplish a limited task that demonstrates capabilities that would normally require a functioning hippocampus. It is a proof of concept, but still less than a full prosthesis for the piece of neural circuitry that it was built to replace.

2. Berger's approach to the development of the prostheses was careful to make few assumptions about the structure and operation of the piece of tissue it was built to replace (they did make assumptions by limiting their analysis to neural spikes). They used a black/gray-box system-identification approach, generating suitable transfer functions for the device from recordings of input and output without asserting constraints such as PRTM would do: 100 nodes, a specific hierarchical manner of processing, etc. The development of the device was therefore open to all possibilities, as long as the result was a device that could fulfill the desired function. As discussed earlier, mapping the signals from this type of implant to a 
PRTM-like interface should probably be implemented in adapters outside of the physical implant.

8) Saving content that was previously "pruned" from the biological neocortex

due to little use of them after having captured them in an already saturated bioneocortex. Such saved pruned content can be reused again for reloading when nonbiological neocortex extension becomes available, e.g. in the Cloud in the MED phase. This is similar to mitigating the pathological conditions in case of pursuing the above objectives but here it is applicable also to healthy brains simply for expanding the capacity of the neocortex in order to reduce the extent of forgetting prior experiences. Prior to the BMI implant supported seamless neocortex-Cloud extension scenario, the implementation of the 'in silico' PRTM POP simulator is similar to some of the redundant configurations used in traditional fault tolerant von Neumann computing network. Keeping a synchronous PRTM POP simulator as stage IV equipment running as a "shadow processing" backup unit in parallel with the 'in vivo' neocortex would support the potential of "swap" or "restore" scenario in an effort to meet objectives 5) 8) here. It would be appropriate to talk about a "swap" scenario, if the reanimated biological neocortex was to loose all of its content and the neural structure itself needs to be reengineered, typically by nanotechnology procedures. Since long years go by between suspension and reanimation, it is typically not a "hot swap" arrangement, although as indicated above, some degree of updates to the backup PRTM POP simulator content could be processed to reflect newly found data impacting the knowledge of what may or should have been stored in the biological substrate at the time of suspension. "Restore" could also be distinguished as cold, warm or hot. Closest to the hot restore configuration needed when the Cloud based MED scenario is already operational and the biological neocortex is already getting damaged by dementia without immediate biomedical repair performed. We do not know, if such scenario practically emerges in the future, given the unknowns in the timing of satisfactory success in biomedical treatments. Closest to the cold restore configuration is applicable to discuss, when the suspension occurs prior to the deployment of the BMI implant based seamless extensions in the Cloud, but the PRTM POP simulator backup is already operational and it is storing biologically lost information due to pathological damage, or due to pruning from saturated neocortex capacity and it is utilizing additional contextual information that the patient was unaware of.

\section{9) Optimize content retention}

and enrichment based on PRTM resource availability estimates, including the availability of free PRTM modules, connections, etc. by preparing a prioritized plan prior to the existence of future extensions, what extra information will be loaded in the extension first. The Cloud gateway BMI implant will negotiate and control the content allocation between the biological neocortex and the Cloud, the extra prioritized 'legacy' information is precompiled by the stage IV device shown in Figure 2 as the PRTM POP simulator. Physically, the simplest architecture is to preload the new extra content from the stage device into the Cloud first, from where the implant can start performing the upload/download operations between the neocortex and the Cloud.

10) Provide feedback to brain reverse engineering,

whether content capturing is feasible based on current assumptions of the brain model. On the one hand, the assumptions of the neocortex architecture consisting of 300 millions 
of PRTM modules of a similarly structured one hundred neurons will be further validated and confirmed by future neuroscience research. From the IT system architecture definition point of view, by applying continuous refinements in the PRTM module allocation expectations; the feasibility of the assumed PRTM neocortex architecture can be validated in terms of how it relates to the IT modeling of the world around the subject's consciousness.

11) Provide input to the design and plan of the neocortex extensions.

Benchmark measurements of storage capacity usage and traffic throughput for PRTM module content allocations when running the PRTM POP simulator in the stage IV unit can be directly applied to specifying the physical requirements for the design of the implant, the relevant in-cloud resources and the communication need in between them.

\section{Integration Framework for External Content Fusion}

The idea of collecting digitized personal experiential data for the purpose of building a digital persona model of individuals as a specific of the 'generic' persona models has been already disclosed close to a decade ago (Seymour L. G., 2005). The idea illustration is shown in Figure 3 below.

\section{Interpret Content - Build Person Model}

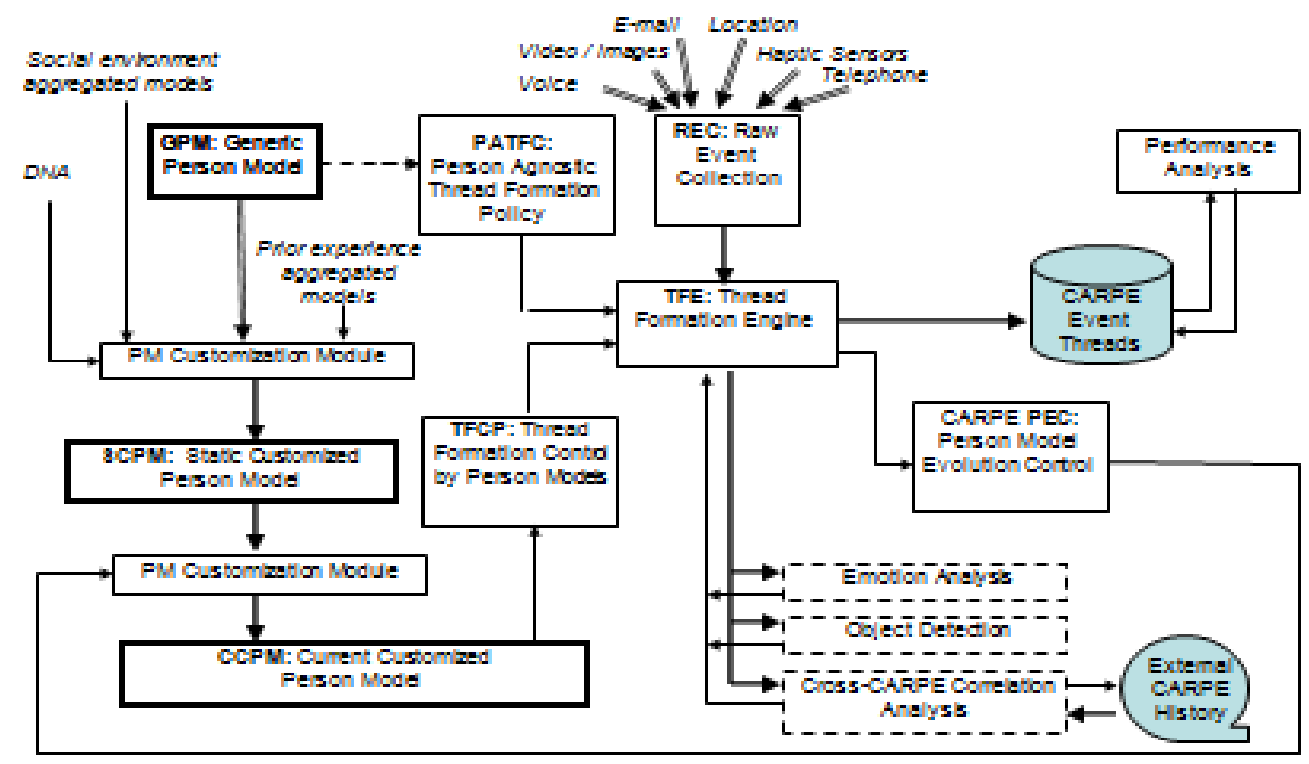

$11 / 11 / 2005$

PersinVitro, MM 2005

\section{Figure 3 Collecting and Integrating Digital Experiential Data Into Persona Model}

Figure 4 illustrates a "plug-and-play" framework architecture of integrating all the components needed for the for the first two stages of the process in Figure 2. The framework builds and 
maintains a PA Persona Agent. It has all the information about the subject that is needed for any kind of commercial service consumption that needs personalization.

PA has four uniform interface planes to communicate with its ecosystem:

- the Southbound plane is for consuming external content stream representing LifeLog data or LifeLog relevant data

- the Westbound plane serves for connecting to analytics modules used for building the PA as a high-level Personhood model, also depicted as Stage II in Figure 2.

- the Northbound plane serves for visualizing PA related data, either for some specific vertical commercial application, or for diagnostic purposes to build a system, such as the solution discussed in this document

- the Eastbound plane serves for connecting to other similar and/or related ecosubsystems for cowdsourcing and for other interoperability purposes. For example, when heart-rate, location and other contextual readings are consumed through the Southbound interface, the Eastbound plane can be used to 'normalize' the person-specific heart-rate measurements relative to a given context and based on the heart-rate measurement values of other people in a similar situation, i.e. context.

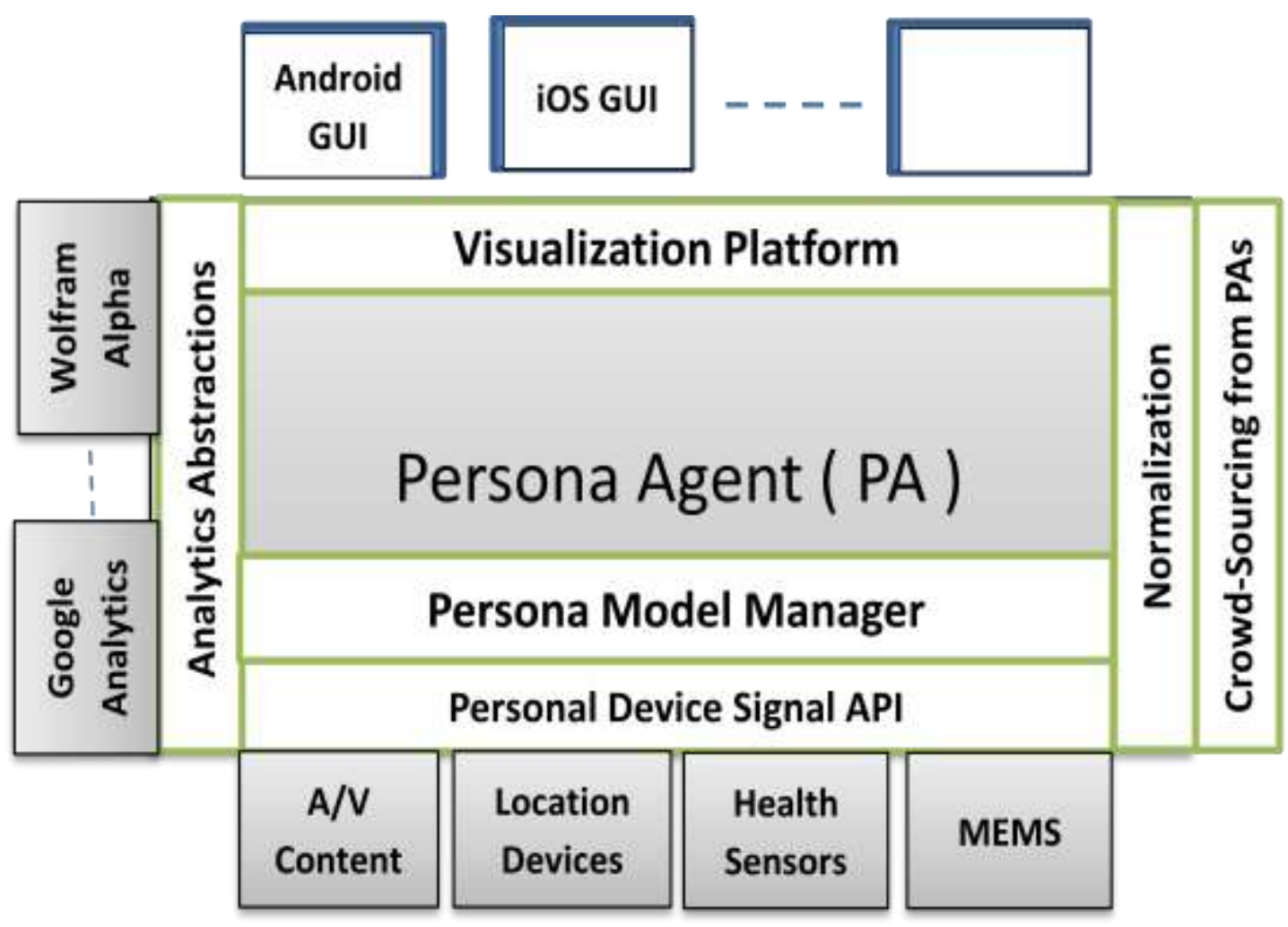

Figure 4 A High-Level Reference Architecture for Collecting, Logging and Analyzing Digitized Personal Experiential Data

The Eastbound plane also serves as a potential gateway to other trusted systems that also maintain data relevant to the Persona Model in this framework. A trusted system 
could be a trusted access to a social network where the same subject is a subscriber. Trusted access can also be provided to similarly situated other persons' PA for subsequent normalization of the person specific device measurements. For example, to determine the impact on the extent of anxiety by reading the heart rate, skin moisture, etc. measurements from a wearable devices of a specific person, comparative analytics across a large number of similarly situated persons provide more accurate ratings, where the similarly situated benchmark pool of people are at the same location and/or at the same time, attending similar events, in similar job functions, and/or are of similar age.

\subsection{Consuming external content stream sources - The Southbound plane}

The external sources include

- Audio-visual data sources, such as a Google-glass, other AR wearable devices, adventure cameras, microphones, etc.

- $\quad$ sensory devices for health and life style monitoring, known from the Quantified Self (QS) community

- communication devices input-output: computers, phones, faxes, e- mail, bills, etc.

- legacy archive of the above

\subsection{Analytics}

Analytics modules are used to map external content stream and their repositories to entities such as

- psychological personality states and traits;

- interests, incentives, focus and objectives;

- conceptual structures: relations to people, places, institutions, communities, events, memes,

Analytics in the framework use standard Semantic Web ontologies to drive these mappings.

A good number of vendors and platforms are available that are in high demand in today's "Big Data" market: Graph Database, Google Analytics, Alpine Data Lab, etc.

Currently ubiqutiously used software specification technologies, UML, RDF and much of the persona semantics work summarized by (Minsky, 2013) are utilized to derive and capture the results of analytics.

In the area of multimedia research and startup efforts, good progess has been made in recognizing classes of objects and concepts in multimedia content. This will be vey important in compiling high-level personhood model data into CMR level data, since some very interesting pogress has also been made in the area of recognizing a similar set of concepts and object types in live neocortex utilizing fMRI. An example of such annotated fMRI image is shown in Figure 5 below, as published on the Web-site of the authors' of (Gallant J, et. al. 2013).

\subsection{Emerging Quantified Self Platform Player Startups}

A number or platform player products have emerged in the Quatified Self startup market: 
- TicTrac - with the primary strength of comparative visualization of several different QS variables from potentially several different device sources on the same screen, so that it would be convenient to correlate them with bare eyes, then intuitively discovering personal dependencies on how these variables impact one another, life-style patterns, behavioral patterns and affective states.

- Singly - pulls data from a variety of Web-based, mostly social network sourced QS data, integrating them for a combined output. Commercially, their focus is on selling to organizations of larger member population, e.g. to employers overseeing employees. Singly would then provide access to employee's derived, summary individual health data. This startup was acquired in 2013.

- Human API - the most recent entrant with a completely open and committed API to a $3^{\text {rd }}$ party development market. They are abstracting the pull of genetic personality traits, location and a half a dozen medical parameters including heart rate, blood pressure, blood glucose, BMI, body fat, etc. 


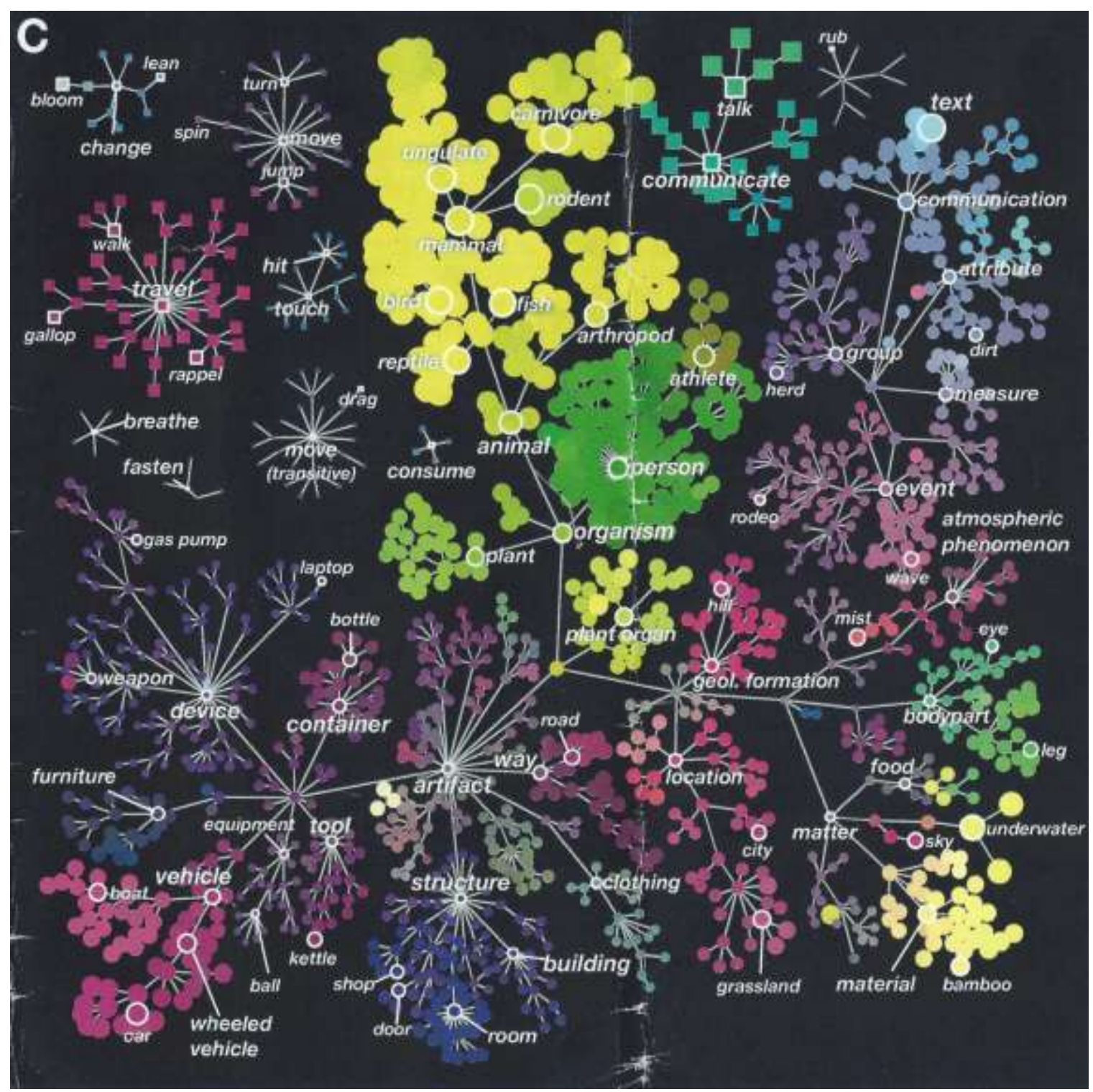

Figure 5 Concepts Represented in the Neocortex Visualized in Interpreted Brain fMRI Images

\subsection{A Framework Implementation Scenario}

An important objective of building declarative consciousness maintenance platforms was an ability to observe and validate the extent of completeness of the lifelog data coverage. All four planes of the framework contribute to architecting such platform.

The first most important selection is the LifeLog content stream consumption API. While all Quantified Self platform suppliers would obviously have such APIs, they are exposing it to a variety degrees at this time.

Since Human API is still open and evolving with a willingness to adopt to the emerging customer needs, it seems the most suitable interface to build on, when constructing the proposed 
architecture for declarative consciousness reconstruction. In the rest of this section, a brief summary of such architecture is provided.

LifeLog experiential content base is marked with metadata referring to value combinations that can be retrieved from the user through Human API.

A "SelfMedia Dashboard" visualization program scans through the content base for matching content components against value combinations of the Human API read-out variables across the entire domain of the implemented Human API. The resulting coverage matrix is visualized using the Dashboard GUI.

For example, the Dashboard would visualize how much content is available in the LifeLog where I exhibit my "extravert" personality traits in pictures, e-mail text analysis, phone call / SMS density; while I am in my office space and my fat content in lowest $20 \%$ of the range for the last 3 years, etc.

Optionally, the Dashboard can also invoke a LifeLog content browser, where clickable Dashboard areas can be expanded into viewing the tagged content itself via multimedia players, text display, etc.

The Dashboard user can edit the Human API read-out value constraints and watch the resulting Dashboard changes. A similar GUI editor can be used to update the "Human API compliant" metadata on the LifeLog content base components through a complement of the Human API layer. An important feature of the Dashboard is clearly visualizing the metadata "white space", i.e. the subsets of the Human API readout variable value space that does not have any experiential content in the LifeLog yet. Shading is employed in the Dashboard to show the "density" of the content availability within specified metadata value space subsets, optionally also visualizing how much of the content is redundant and from what point of view.

The resolution and richness of the semantical definition of the LifeLog content can be further enhanced in the future with options applying tools such as Graph Lab database and the underlying RDF definitions to analyze semantical connectivity between LifeLog components while feeding the Dashboard visualization with coverage strength data.

In order to support a variety of marketable niche applications, where the ability to map to a neocortex architecture representation is not important, the framework also supports a configuration function. The configuration option of the "SelfMedia Dashboard" screens can carve out subsets of the entire experiential content based on satisfying constraints defined on the Human API readout variable value space. The carved out content subsets can be copied and transferred to the user space of the application, subject to their access right. Another option of the configuration is based on a user access subscription management registry, where each user is assigned a Human API constraint definition. Within such constraints, each subscriber can access all or limited Dashboard browsing features in real-time.

Another potential option is using extensions of the Human API implemented by pluggable analytics modules to provide additional metadata that can be harvested from the available experiential LifeLog content itself, for example stress level measurements derived from the captured multimedia content itself - as opposed to directly from biosensor devices. In this way, recursive extensions of the "Human API" can be implemented, where analytics can be invoked either directly from the Westbound interface plane, or, analytics can be performed externally to the framework and the externally derived analytics results as additional persona attributes can be fetched through the extended Human API from the Southbound interface plane. 


\section{The Mapping Process}

In this Section the mapping process is described in more details showing how the LifeLog based specifications are used to assist modeling the brain at the PRTM architecture level.

\subsection{Functional Decomposition of the Consciousness Data Processing Flow}

Figure 6 below illustrates a more detailed functional decomposition flow of the higher level consciousness data processing flow outlined in Figure 2 using incremental LifeLog data compilation.

The inter-functional relations in this model fall into several categories and they are depicted with different line styles in Figure 6:

- Consciousness data content flow

- Operational control impact between functional entities

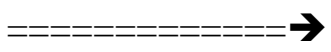

- Specific subject consciousness model update impact - - - - - - - - ->

- Impact on technology component design or on investigations ._._-_-_..->

The numbers assigned to these relations in Figure 6 signify the objectives described in Section 6 Detailed Objectives, thus illustrating the coverage of these objectives by the proposed mapping process architecture here. The notation used in Figure 6 to differentiate between the time-frame of developments of the data and functional entities shown in Figure $\mathbf{6}$ are explained in Figure 6.1.

Four domains of functional components are identified here:

1) LifeLog data collection domain -

This is Stage I in the process shown in Figure 2. A typical reference instance of this domain was the Microsoft MyLifeBits research program (Bell G., 2004). For purposes of consciousness reconstruction, the Terasem Foundation's "Cyberev" tool set can serve as an operational state of the art system. The output of this domain are minimally processed raw measurements collected and produced by electronics devices for multimedia capturing, quantified self type of sensors. This output is labeled as MLB (My Life Bits) in Figure 6. The contextual metadata here is minimal, including time and location stamps and the ID and type of the collecting device. Harvesting and feeding legacy LifeLog data is also included in this domain, such as scanning old photos, letters, etc., retrieving old e-mails, etc. Certain device and raw signal carrier specific preprocessing is also included here to unify the semantical clusterization and recognition functions in Domain 4 below. For example when "Human API" is providing heart rate data stream, the data representation is independent from the make of the wearable device collecting the measurements.

2) Biological neocortex PRTM domain -

This domain is expected to be under development until the seamless MED milestone described in (Kurzweil, 2012) is reached. The functional entities of this domain include the

a) The biological neocortex 
b) The MED BMI “implant” (Kurzweil, 2012)

c) The extension of the neocortex in the Cloud

d) The on-going "brain reverse engineering and mapping" computational neuroscience research process

e) Live fMRI and other brain scan result data instance. We call it 'instance' here, because similar data could also be part of the LifeLog data measurements. The use of the two different instances is for different purpose and in different context. Here, this is for the support of the brain reverse engineering research in general. In the LifeLog domain, it can provide important metadata of the other raw LifeLog measurements and experiential content, specific to the life and person model of the subject. For example, electromagnetic signal measurements could indicate stress level that can be fused with other indications of stress level, such as functions of differentials of heart rate values. In other examples, this could be used as a metadata to indicate focus on certain fragments of multimedia experiential content.

f) Existing AI/AGI knowledge base system, such as the ones supporting Watson, Siri and others. These can also be perceived as being used in different instances in two different domains. They do support the brain reverse engineering research in general by providing a more comprehensive understanding of the functional behavior of the human brain in a variety of use case analysis investigations. Their functionality as platform is also necessary to process the LifeLog output content for their semantical interpretation both in the context of other LifeLog data of the subject and in the context of public encyclopedia data.

\section{3) Supplementary brain preservation disciplines domain.}

Before the emergence of LifeLog technologies and computational neuroscience, a few brain preservation efforts emerged mostly based on advancements in biomedicine. The most commonly known is Cryonics. Up until most recently, these were viewed as orthogonal to and independent from the first two domains above. In the last few years, it started changing, as illustrated in Figure 7 and also promoted by the authors of (More, M. 2013) and (Rothblatt, M. 2012).

4) Incremental cross-compilation IT domain.

This domain is the "bridge" between the above first two domains implemented as an incremental compilation process through two intermediate representations of the human consciousness data, also depicted in Figure 2 as stage II and III. This domain is the focus of the efforts targeted in this publication. Several functional entities are included in this domain as described below.

\section{a) Incremental data compiler from MLB representation to CMR representation.}

This is equivalent to the Stage II processing component in Figure 2. Internally, it produces and maintains a higher-level, semantically rich representation of consciousness data shown as "Personhood high-level model of consciousness" in Figure 2, derived from the first stage MLB data by analytics and these were represented in the first two layers of Figure 1. More detailed description of the data at this level was provided in Section 5.2 What is a Persona Model? 
b) CMR format repository of the consciousness data.

This is the Stage III representation of neocortex specific data in Figure 2 but still abstracted from different detailed computational neuroscience approaches of modeling the neocortex. This function is the repository of the data produced by the incremental compiler in function a) above.

c) MMI interactions with the incremental compiler.

The function managing these interactions is shown as "Diagnostics \& Control" in Figure 6. Other than laboratory experimental platforms, MMI interactions can be feasible in additional commercial products built on the same platform and having a monetized, visible, main value proposition function harnessing some or most of these MMI interactions. In this case, the incremental compilation support functions for consciousness modeling progress are passively harnessed, i.e. not necessarily distracting the subject user. The potentially included such support functions are listed below.

i) Completeness analysis results can be visualized using this interface as outlined in the "Dashboard configuration" description of the illustration in Figure 4 above.

ii) Semantical ambiguities can be manually resolved here, as an option.

iii) This is also the interface to exert optional, additional control on what public contextual information should be harnessed to provide richer semantical context of the MLB data.

iv) Conversations with the subject can also be optionally planned through this interface with the result fed back here for further semantical analysis, metadata enrichment and resolution of more, outstanding ambiguities.

Diagnostics techniques are needed here that would keep the disruptive side-effects and cost of these supplemental interactions to the minimum. These are described in more details in Sections 8.2.

d) External knowledge base supported semantical analysis system. This is a knowledge base similar to ones used in commercial and known research programs, such as Siri, Watson, etc. Analytics toolsets, Google Analytics, Graph Lab database, Alpine Data Lab, etc. are also provided with this function. This are tools and KB to support functions listed above in a) and c).

e) CMR data repository analyzer supporting the incremental compiler. This function can collect benchmark measurements of the scope and simulation statistics of the CMR representation of the neocortex. The benchmark data is fed back to the incremental compiler to assist in the semantics analysis, optimize the compilation process and prioritize the output of it. For example, if the neocortex is significantly saturated while much of the stored data is significantly aged, the likelihood of pruning them is increased and this information could be valuable of the semantics 
analysis in for the incremental compiler since there is a significant likelihood that the subject may have ignored some important implications of fresh events.

f) CMR PRTM format simulator, running continuously to support the incremental compilation through the CMR analyzer.

g) CMR format downloader from the CMR repository to the neocortex extension Cloud. Operationally, the download here is close to the arrangement used at the time of and after the seamless MED BMI implant is deployed. But even earlier, a simulator of the implant can be built to prepare for the first live download via the MED BMI implant. The simulated implant is not illustrated in Figure 6, but it could be interacting with the CMR simulator and with its Cloud extension using an interface protocol similar to one deployed in the future for the MED embedded implant. 


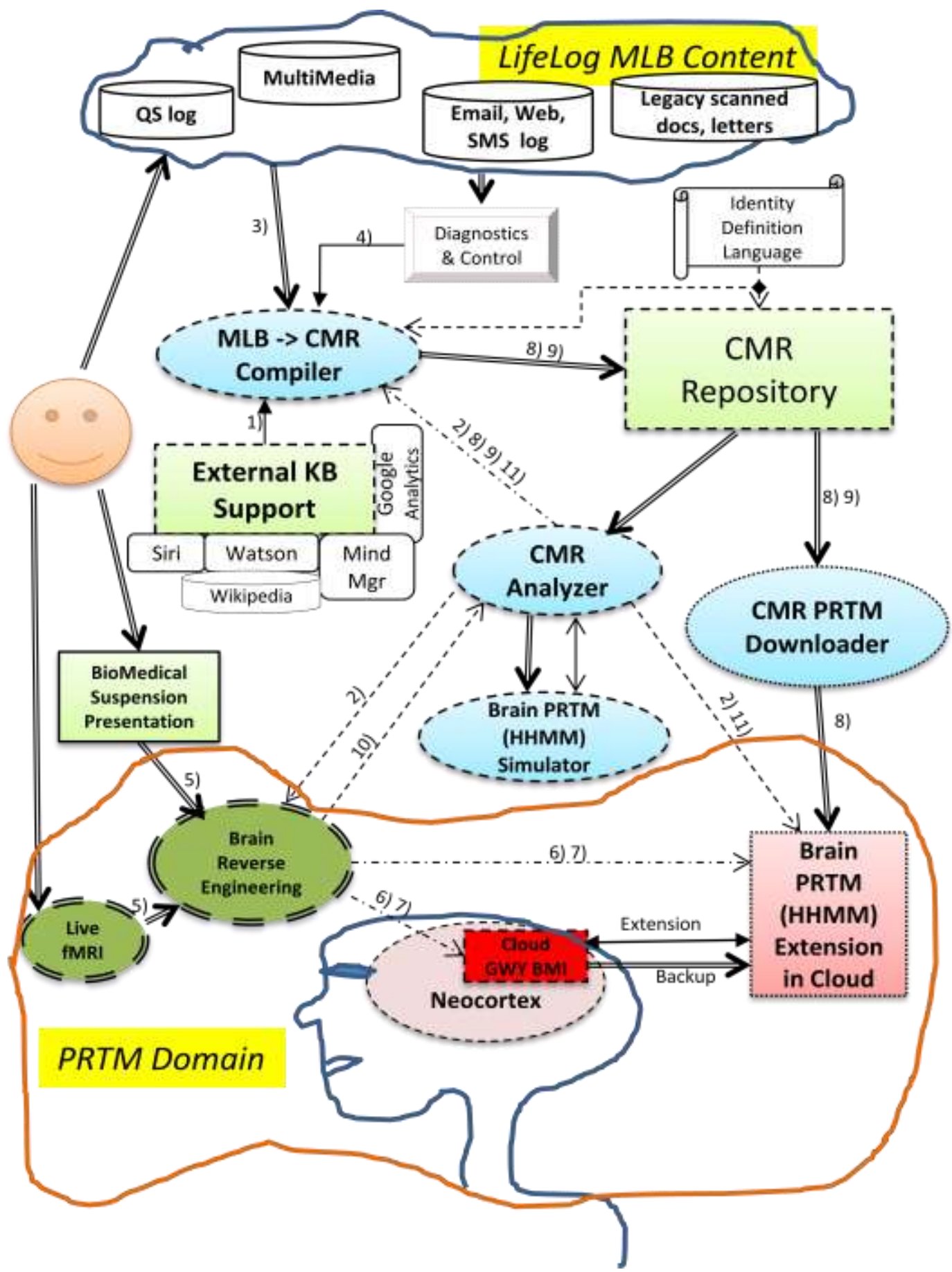

Figure 6. Bridging LifeLog Content Repositories and Brain Structure Using Analytics Based Incremental Content Data Compilation

The notation used to identify the timing of potential availability of the data and functional entity components shown in Figure $\mathbf{6}$ are illustrated in Figure $\mathbf{6 . 1}$ below. 
Existing technology content

Content format with technology development that can start at this time

Content format with technology development that can start after MED (Mind Extension Deployment) is matured

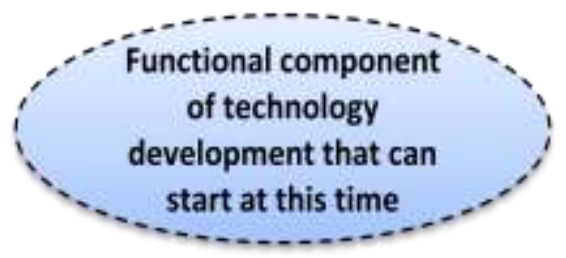

Functional component of technology development that can start after MED ( Mind Extension Deployment ) is matured

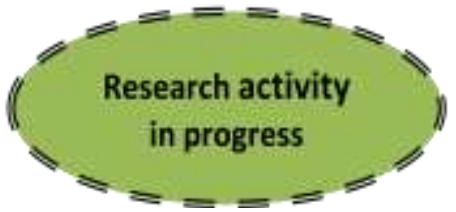

Figure 6.1. Notation used to identify the timing of potential availability of the data and functional entity components

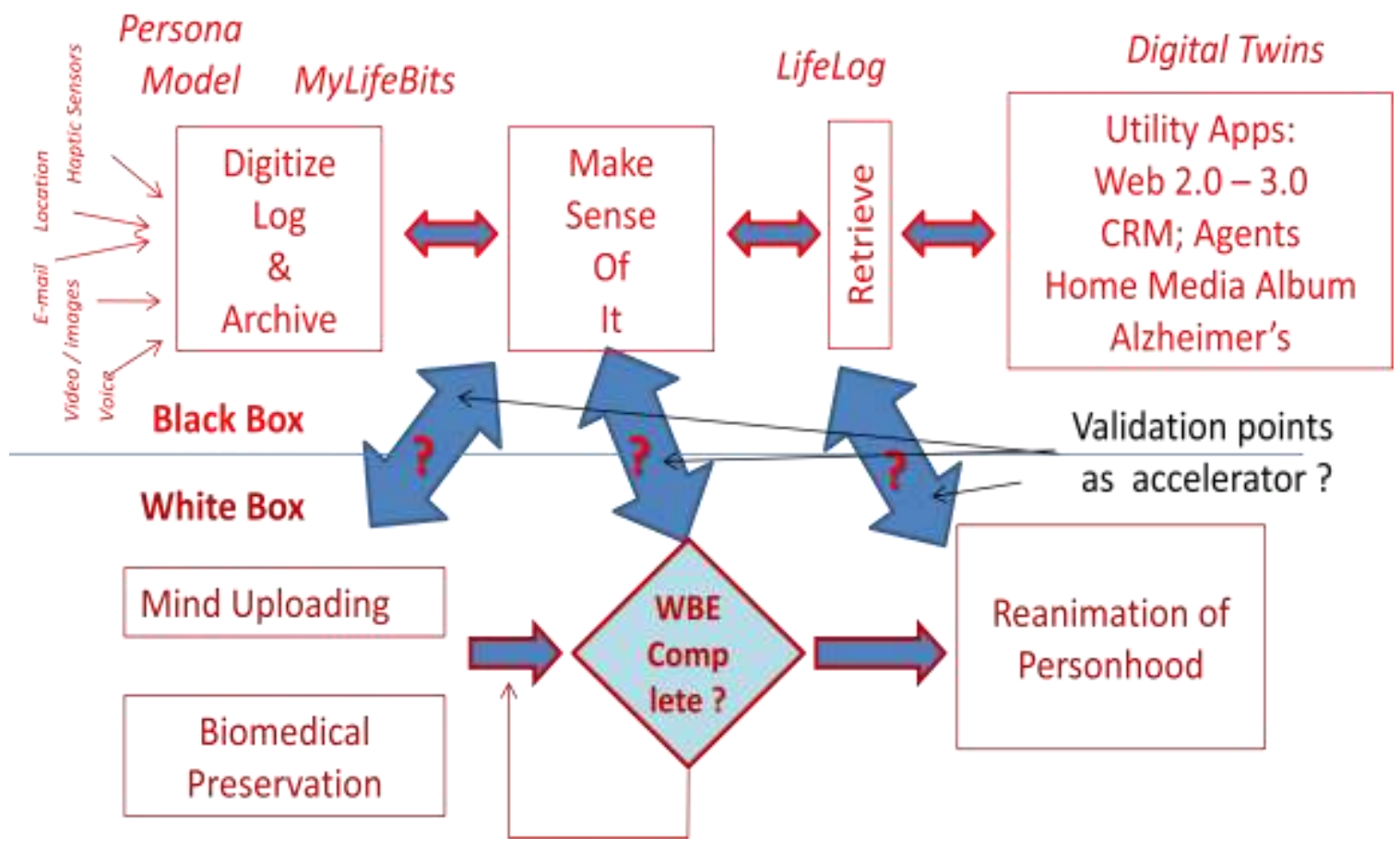

Figure 7. An early illustration of the concept of using white-box model validation by black-box models as presented at the Nano-Bio-Cogno Convergence'08 Unconference 


\subsection{Minimizing the Cost and Disruptive Impact of Diagnostics Dialogs}

The diagnostics and control interactions supporting the build-up and conversion of the MLB persona model illustrated in Figure 6 will have to deal with two types of challenges: the cost of inserting new dialogs with potentially duplicating previously recorded content; and potential behavioral changes as side-effects of conducting such diagnostic dialog.

\subsubsection{How to Minimize the Active Diagnostic Communication OVERHEAD?}

The question here arises how much of the recorded input and output data would have to be embedded in the diagnostics dialog. The goal is clearly to have make such dialog as passive as possible, since this has been a long-standing objective of all LifeLog technology solutions.

The dialog designer engine's intelligence would rank the importance of validating recollections of past experiences and/or the resulting behavioral impact. Just by monitoring the content produced by the subject and by meeting Objective 3), it can be implicitly validated that the data has actually been retained in the neocortex. Another factor in the prioritization is the ambiguity of such implicit storage determination. There will be implicitly though but clearly and highly suspected "white-spaces" in the recorded content coverage, that did not show up as constituents of the outbound content, although it should have been there based on the semantical coverage of the previous dialogs. Yet another important priority weight factor is the urgency of the clarification if a piece of previously recorded information or its impact is present or not, in view of the need for clarification of the contextual factors of new input record to be analyzed. Similar urgency can also be justified by reaching certain suspected neocortex saturation thresholds and the related need to determine, what got discarded to make room for the new experiences. Such scenario could be likely after high-impact, disruptive events in life, especially after early youth, when the neocortex tends to get saturated ( Kurzweil, R. 2013 ). Yet another priority could arise as a result of ambiguities in interpreting recent brain imaging results, where such disambiguation could efficiently lead to a higher volume of indirect disambiguation of related content storage.

\subsubsection{Diagnostics Impact ON THE PeRSONALITY CHANGES.}

Using the prioritization techniques described in the previous section, the diagnostics engine intelligence could reduce the need for explicit inquiries to the minimum. Another technique to ensure the maximum use of "passive" inquiries is combining such diagnostics engine functionality with commercially needed applications serving the person's needs in regular life. In other words, when selecting tools for making the subject's like more productive and enjoyable. favoring brands of software and computer products that are "persona model building friendly", they comply with APIs to share maximum amount of data with the diagnostics engine. Even if the use of explicit diagnostics inquiry is unavoidable, the input and response could also be recorded and analyzed just like any other LifeLog content record. In this sense, the way life is lived would be impacted to some extent, but it should not be radically different from the impact of using new and disruptive technologies in the past.

\subsection{Technology Process Specification and Implementation Detail Issues to Address}

This Section describes the detailed issues that need to be addressed in the subsequent phases of the development process, in order to implement the solution of meeting the objectives as illustrated in Figure 6. Four phases of implementation design and detailed specification issue resolutions are outlined below. 


\section{DECLARATIVE CONSCIOUSNESS FOR RECONSTRUCTION}

\section{a) Phase A}

i. Determine what semantics modifiers need to be added to the LifeLog ontology based content in the stage II "personhood high-level person model" of the compilation, in order to sufficiently support the steps of deriving the

1. PRTM module connections,

2. weights, inhibitors/amplifiers in axons, dendrites,

3. concept object framework patterns of images, events, memes, etc.;

4. separate inherited patterns: what other related objects, driven by the results of the Berkeley fMRI maps (Gallant, J. et.al. 2013) are inherited per persona types, and per all humans;

5. 'size' parameter (e.g. phoneme length), as specified in (Kurzweil, 2012);

6. age of pattern (Kurzweil, 2012);

7. occurrence statistics, in order to facilitate decisions such as what concepts will be most and least likely to be pruned from the neocortex after it gets saturated, what goes to the Cloud and what stays in the biological neocortex, etc. as detailed in Phase b), details i.a.i and i.a.ii.

ii. In the timeline between 2013 and the date of a full MED, at what point should be contextual LifeLog metadata added to the consciousness data that ultimately need to be captured in CMR format? There is a two-way dependency between this decision and the factors listed above. Performing it right after collecting the raw sensory measurements in stage I of the process shown in Figure 2 would benefit from the higher probability of the availability of many temporal contextual factors. Performing it later based on a forensic context mining, around the time of deploying a MED BMI implant, would benefit from the availability of a superior AI technology of deriving and relating nearly all possible by that time contextual factors. A combined use of both methods seems likely to be the most effective solution.

iii. What are the specific available KB tools and content from commercial, open source or otherwise accessible sources that could be utilized for the incremental compilation semantics analysis and PRTMM content mining support uses, such as the KB ecosystem fusion, NL interpretation, Q\&A conversational MMI, etc.? Candidate tools include Siri KB , Watson, WolframAlpha.com, etc.?

iv. What are those limited CMR simulation scenario choices, where sets of PRTM modules can be stubbed out in order to remove them from the participation in the event (thread) pattern recognition processing? These are important decisions impacting the progress in the development, since feedback can be generated long before a full brain emulation capacity is achieved by indicating what will be missing in different passes of the incremental CMR compilation process.

v. What are the detailed components of the MMI conversation to identify items that the subject was unaware of, content shared across persona types, impacting weights, etc?

vi. What security and privacy protection constructs should be added to the Stage II and to Stage II level data? What are the envisioned policies that would likely to take advantage of these constructs? These may not have to be developed in the early stages 
of the data representation detail definitions, but the early public concerns related to the use of implants and uploading in this area would justify the design steps. At the Stage II level, classification tags should be considered for nodes in the semantical nets, for restricting the use to certain substrates, for types of content, for levels in the neocortex PRTM module hierarchy, etc. These would also enable the Stage II browsers and dashboards described in 4c) for Figure 6 to visualize how open/restricted is the access to a variety of scope and type of content.

\section{b) Phase B}

i. What neocortex layers will be in the Cloud-based extension? In general, in the highest layers are the identity/self-awareness type of concepts, while in the lowest layers the raw sensory data front-end processing. How can we model the different partitioning scenarios on performance impact prior to deploying the MED BMI implant?

ii. What happens with the neocortex patterns that we forget? Can we mark somehow the CMR representation content parts with the likelihood of being forgotten, while the solution is still not deployed with the embedded implant in the brain? Can these markers control somehow the CMR download process and use predetermined priorities how to partition the different segments, embedded in brain vs. Cloud based? Can we deal in this solution with scenarios such as DéjàVu, dreams, Freudian sub consciousness, and similar ambiguities?

iii. At what time should we run CMR conversion preprocessing for download? Should it be done as soon as possible, while having a younger mind, or just before the PRTMM load when the technology is superior, but potentially has to deal with defective mind effects?

iv. Consider compression methods to apply in Stages I, II and II: for multimedia, image and sound, use contours only, as described in (Kurzweil, 2012); make use of inheritance redundancy ( e.g. persona type ) by applying pointers only vs. keeping details redundant across persons to repair potential defects in neocortex, gateway communication, or in related backup.

v. Detail more formalized scenarios of the N-Phases into CMR compilation: as new external contextual data or brain PRTM architecture feature is discovered; new scanning method is available for LifeLog data creation. In many of these new scenario cases, running another pass on compiling the CMR file would make sense to benefit from adding new 'metadata' helping to map into PRTMM structures of higher fidelity levels.

\section{c) Phase $\mathrm{C}$}

i. (Kurzweil, 2012) described a suggestion of repairing "holes" in the neocortex (Chapter 7, pg 176), which could be a result of a pathological condition such as dementia or simply a behavioral side-effect. Using the CMR compilation method described here, there will be an option_of repairing it, or leave it as is (It is me!) and keeping the "patch" in the CMR repository stage III and/or IV shown in Figure 2. in case the owner-subject of this model control process changes his/her mind.

ii. How should the compilation from Stage II to Stage III deal with the subjectivity of contextual interpretation and often reductionist simplifications ( see ( pg 217, Chapter 9 in (Kurzweil, 2012))? Should it store multiple, different, perhaps conflicting contextual 
interpretations of the same Stage I and II logs and high-level models in the Stage III CMR mind of the subject? Then, in a later compilation phase, when more data is available on what got actually captured in the biological neocortex, the conflict could be resolved and only this one alternative would persist for Stages II and IV. Alternatively, several other scenarios could also be simultaneously maintained as alternative possibilities, computational resources permitting. These could support special, additional decision management analysis application aspects, e.g. "what else might have happened in my life, should I have taken a different course at evaluating certain events?".

\section{d) Phase D}

i. How easy it is to map the PRTM based CMR definition to other types of brain modeling representations listed in the section "Existing Research Efforts" and what is their significance?

What are the risks of neglecting the impact of lower-level neural details in the CMR level abstractions, such as the dendritic tree structures as hierarchical computational devices? A more detailed clarification of these concerns are elaborated in Section 8.4 Risks of Choosing Too High-Level CMR models. below.?

ii. What is the exact mapping architecture from the neuron to Cloud PRTM concept modules? This issue is elaborated in more details in 8.3.1 How does the neuro to BMI implant to Cloud connection work with unknown neural structures underneath?

iii. How to apply a PRTM KB supporting the incremental consciousness data compilation here also for advising the subject prior to and around the BMI implant based MED on the trade-offs in using the BMI implant? Given the history and the prognosis of the speed of improvements in the fidelity level and in the scope of the PRTM model coverage, how does the risk of injury weighs against the risk of loosing irrecoverable consciousness defining information?. ( (Kurzweil, 2012) pg 247, Chapter 9 )

iv. How good the fidelity levels of the backup PRTM models should/could be? What quality parameters could/should be specified and tracked?

v. How to support the gradual transition of the simulated Stage IV CMR presentation into the extended biological neocortex, such that early sign of unmanageable disruptive aspects of a full immersion would be detected in time for coming up with a mitigation strategy. Such potential disruptions are currently anticipated in the area of perception of security and privacy, unusual contextual changes such as sudden awareness of a broader scope of past event recollection, response time differences when extension is involved, e.g. response time variability due to cloud network dynamics. For assessing some of these in simulation, brain sections beyond the neocortex might also be modeled.

\subsubsection{HOW DOES THE NEURO TO BMI IMPLANT TO CLOUD CONNECTION WORK WITH UNKNOWN NEURAL STRUCTURES UNDERNEATH?}

The BMI implant package talks PRTM in an API wrapper in the in silico environment, whether it is a simulator or a Cloud based extension. There is a must have aggregation mapping between neuron level signals and the PRTM module interface. As indicated in Section 5.5., this mapping 
function may not be fixed for all modules in the entire neocortex, across individuals, and it may not be permanent either.

The Berger implant is viewed as a VLSI channel between the short term memory and the long term memory. To make it a BMI, it needs wireless connection to a receiver placed on/around the body as a wearable. The digitized EMF signals produced by the receiver in the wearable are decoded to support the PRTM API. This decoding is viewed as potentially quite intelligent, since the EMF code is likely to be needed to identify what concept was represented in the PRTM module inducing the EMF signal.

More details here would have to be based on further studies, e.g. how the Gallant lab is rendering cell areas to concepts. The mapping between the biological neuron ID and the concept ID would have to be implemented in a distributed architecture, the high-end integration of it would be definitely in the cloud, some low-end caching could even be in the BMI implant. The bulk of the mapping implementation has a hierarchical distribution across wearable, an optional, personal gateway between the wearable and cloud, and the persona agent in the cloud.

\subsection{Risks of Choosing Too High-Level CMR models}

Builders of IT persona models should appreciate, that the currently known and listed in Section 4 sources of the CMR-level definitions are not low-level but sometimes even considered as too high-level abstractions from the computational neuroscience point of view. For example, HTM in (Hawkins J, et.al. 2011) is already quite abstract for neuroscientists and several levels or reinterpretation removed from lower-level models such as one would find in the Blue Brain program. In fact, some critiques of HTM stated that it had possibly already discarded important details of the neuroscientific models and might no longer correspond to a reliable neocortex model. An important point to consider is that Kurzweil's approach, as well as the HTM model completely neglect the significance of dendritic trees as the hierarchical computational devices of true neuronal circuitry. Perhaps an abstract representation in terms of neuron "nodes" can replace those devices, but the mapping has never been shown convincingly in either HTM or the model proposed in Kurzweil's recent writings.

However, in order to make progress in a unified mapping from the LifeLog constructs, it is proposed to stop at the CMR level here. It should be explicitly mentioned in the CMR level description, that at this level, we can not guarantee that no further modifications will be needed to this model, as we become more aware of more differentiators below the CMR levels that can not be abstracted by the current CMR specification. As it is illustrated in Figure 6, we need to start building simulators and analyzers at the CMR level and we need to start building highly resource intensive incremental compilers from MyLifeBits level content to a relatively stable "brainmachine" principles of operation" architecture. It is exactly a main purpose of this IT experimentation program to discover gaps between the experiential behavior of the subject at the LifeLog content level, while modeling at the "highest common denominator" CMR machine architecture use vs. using a more specific and lower level neural structures as a machine-level execution platform. . If the difference is not significant, or can be accommodated at the CMR interpretation functions without much difficulties, very significant brain storage capacity can also be saved in all substrates and in all phases of the program. 


\section{Next Steps}

Before resolving the above outstanding issues, the currently existing technology landscape assessments for the four domains in Figure 6 need to be completed: for LifeLog data collection, for the biological neocortex domain, for the supplementary brain preservation discipline domain, and for the analysis of LifeLog data to create personhood model. The assessments need to focus on the needs of the missing parts of domain 4). In particular, the issues listed below will need attention.

A. LifeLog data collection domain. This is the Stage I domain in the process shown in Figure 2. Current business ecosystem landscape needs to be described: QS/LifeLog startups, social networks for data collection, etc. A good number of them are listed in various Facebook and LinkedIn special interest groups, such as Carboncopies, Quantified Self, Reconstructive Consciousness, and others.

B. Biological neocortex domain.

- Kurzweil's vision of the BMI implant based MED in the Cloud;

○ technology challenges of the gateway implementation, resolution, access to neocortex module data at all levels, magnetic radiation impact.

$\circ$ what could be crowd-sourced and then shared across personal neocortex concept clusters

C. Supplementary brain preservation disciplines domain

This is an assessment of technology progress made in cryonics, chemical brainpreservation and to some extent, in archeology and anthropology, as it may relate to relevant information storage in the biological substrate of subjects after pronouncing their legal death.

D. Incremental Cross-compilation IT domain

- Neocortex inferred internal state simulation:

- reference baseline: currently Berkeley Gallant-Lab fMRI object map;

- how does the above map compare to visual object classification recognition in leading edge research and marketable startup efforts?

- separation of aware and unaware sets of objects and events in the high-level model. How do these change in time?

- How exactly objects are represented in the PRTM POP declarative language

- What is the impact of the PRTM machine having separate operative and backup memory, while swapping content in and out is driven by the external stimuli ?

○ Shorter- term feasibility investigation steps:

- passive "unsupervised" content collection, augmented with conversational guidance;

- determination of awareness boundaries and white spaces 
- storytelling recording and analytics with incentives as a "teaser" application

- conversational Siri-like NL support by GraphDatabase and DashBoards

- comparison with Neocortex state simulation - state update and reinterpretation loops;

- visual concept browsing guidance, incentive and semantic clarification

- how does the definition of a CMR language and representation impact the ease of mapping into it from the Personhood data model?

- startup ecosystem landscape description

- what are the market incentives to turn such MyLifeBits++ solution into product ? For example, could "process compliance metrics" on how organized your life is ever become a marketable product ? What is the LifeLog completeness and fidelity-level of your data in LinkedIn, Facebook, Cyberev, etc? Could these become interesting questions with business value attached to the answers?

\section{Conclusions}

- "Reverse engineering the brain", "understanding consciousness", "mind uploading", "brain preservation" and other similar objectives can not be accomplished without being able to define successfully what the consciousness of an individual is

- A successful consciousness definition is declarative, formalized and transparent of the brain model definition. It is successful, if the completeness of the definition is measurable, the corresponding content can be mapped into a PRTM-compliant CMR and then, it can be simulated/emulated.

- Formal software specifications methods and tools are the only ones, that have been proven successful in implementing complex systems. Since simulation, emulation and future upload/download are among the envisioned usage scenario, "end user friendly" software specification and design methods and processes need to be used also for consciousness definition where the domain expertise is simply the knowledge about people's life and identity.

- Top-down or bottom-up specification methods only will not work. As a minimum, we need to assess completeness at the top level. We also need the bottom level, so that we know that correct mapping does exist. Right now, we do not have any of these two defined in final form. The "middle-out" approach is the best bet, while the middle level consists of the abstract, high-level self-model representation, the CMR format and the mapping in between these two. Obviously, these two are iteratively refined and thus driving the definition engineering process at all levels.

- This is not a process of pure reverse engineering of the brain content. This is roundtrip engineering. Otherwise, we can not really measure the progress. 


\section{Acknowledgements}

I would like to express my genuine thanks to the reviewers of this paper for recommending numerous observations, clarifications and comments to make it acceptable for publication in the Special Issue of JAGI devoted to the Convergence of Computational Neuroscience and AGI.

\section{References}

Balston, R. M. 1993. Cellular Radio Systems. Boston, London: Artech House.

Bell, G. J. 2004. The Microsoft MyLifeBits project. In Proceedings of CARPE Workshop 2004, ACM Multi-Media Conference 2004 , 48-55. New York,: Microsoft Research

Berger, T.W.; et. al. 1995. VLSI neural network implementation of a hippocampal model. In Proceedings of IEEE International Symposium on Circuits and Systems, ISCAS'95, 16641667 vol 3. IEEE Conference Publications

Berger, T. 2013. Engineering Memories: A Cognitive Neural Prosthesis for Restoring and Enhancing Memory Function. In Proceedings of The Global Futures 2045 International Congress, GF2045, 51 - 61. New York. 2045 Strategic Social Initiative

Chana De Wolf, M. 2013. Reanimation Research Progress and Challenges, Workshop on Cryonics Reanimation Developments. Portland.

Dunin-Barkowski, W. 2013. Current State of the REBRAIN 2045 Brain Reverse Engineering Project. In Proceedings of The Global Futures 2045 International Congress, GF2045, 24, Abstracts. New York. 2045 Strategic Social Initiative

Goertzel, B.; et. al. 2013 Building Better Minds: An Architecture for Artificial General Intelligence.

Eliasmith, C.. 2013. How To Build a Brain - A Neural Architecture for Biological Cognition New York, Oxford University Press.

Hameroff, S. 2013. How Human Consciousness Can be Uploaded via Quantum Teleportation. In Proceedings of The Global Futures 2045 International Congress, GF2045, 26, Abstracts. New York. 2045 Strategic Social Initiative

Hawkins, J; et.al. 2011. Hierarchical Temporal Memory, Technical Report, Version 0.2.1, Numenta, Inc.

IPTO, D. 2003. LifeLog Proposer Information Pamphlet. Solicitation Number: BAA03-30 DARPA IPTO.

Gallant, J.; et.al;. 2013. Attention During Natural Vision Warps Semantic Representation Across the Human Brain, Nature Neuroscience, 76. 763-770

Carmena, H.; and Maharbiz, M. 2013a. Brain Control of Prosthetic Devices: The Road Ahead. In Proceedings of The Global Futures 2045 International Congress, GF2045, 25 - 32. New York. 2045 Strategic Social Initiative

Carmena, J.M.; et.al. 2013b. Neural Dust: An Ultrasonic, Low Power Solution for Chronic Brain-Machine Interfaces. Cornell Uniersity Library 
Kaplan, A.; Lebedev, M.; and Berger, T. 2013. Roundtable on Life Extension of the Brain in a Full Body Prosthesis with Biological Blood Substitutes and Brain Computer Interfaces with Optional Neuroprostheses. In Proceedings of The Global Futures 2045 International Congress, GF2045, 14, Abstracts. New York. 2045 Strategic Social Initiative

Koene, R. 2012. Mind Transfer: Human Brains in Different Materials. New Scientist, 2888

Koene, R. 2013a. Towards a New Strategy for Human Evolution,. In Proceedings of The Global Futures 2045 International Congress, GF2045, 9 - 16. New York. 2045 Strategic Social Initiative

Koene, R. 2013b. Whole Brain Emulation: Reverse Engineering a Mind,. In Proceedings of The Global Futures 2045 International Congress, GF2045, 77 - 88. New York. 2045 Strategic Social Initiative

Kurzweil, R. 2012. How To Create a Mind, London, England, Pinguin Books, Ltd.

Rothblatt, M. 2013. The Goal of Biotechnology is the End of Death. In Proceedings of The Global Futures 2045 International Congress, GF2045, 19, Abstracts. New York. 2045 Strategic Social Initiative

Minsky, M. 2013. Facing the Future. In Proceedings of The Global Futures 2045 International Congress, GF2045, 18, Abstracts. New York. 2045 Strategic Social Initiative

More, M. 2013. Use of Lifelog in Reanimation, Workshop on Cryonics Reanimation Developments. Portland.

Oren, T.I. 2003. Personality Representation Processable in Fuzzy Logic for Human Behavior Simulation.In Proceedings of the 2003 Summer Computer Simulation Conference, 1118. Montreal, Canada: University of Toronto.

Sandberg, A.; and Bostrom, N. 2008 Whole Brain Emulation. A Roadmap, Technical Report, \#2008 - 3, Oxford, England: Future of Humanity Institute, Oxford University.

Seymour, L. G. 2005. Why and How CARPE Should be Personal? In Proceedings of 2nd ACM Workshop on Continuous Archival and Retrieval of Personal Experiences, CARPE '05, 45-52. New York, ACM.

Snyder, M. 2012. The Role of Omics in Computational Biomedicine. IEEE New Frontiers In Computing, Medical Applications. Palo Alto, Calif. IEEE, Stanford.

Snyder, M. et.al. 2012. Cell. 148(6): 1293-1307.

von Neumann, J. 2000. The Computer and the Brain, Second Edition, Mrs. Hepsa Ely Silliman Memorial Lectures.

\section{Glossary}

AAA- Authorization, Authentication and Access control (Balston, D.M. 1993)

BMI - Brain Machine Interface

BMI - Body Muscle Index

CARPE - Capturing, Archiving and Retrieving Personal Experiences (Seymour, L. G. 2005)

CMR - Canonical Mind Representation 


\section{DECLARATIVE CONSCIOUSNESS FOR RECONSTRUCTION}

CRM - Customer Relationship Management

EMF- Electro-Magnetic Field

fMRI - field Magnetic Resonance Imaging

GSM- Global System for Mobile communication (Balston, D.M. 1993)

GWY- Gateway

HLR- Host Location Register (Balston,M. 1993)

HMM - Hierarchical Markov Model (Kurzweil, 2012)

HHMM - Hierarchical Hidden Markov Model (Kurzweil, 2012)

HTM- Hierarchical Time Model (Hawkins, J. 2011)

IT - Information Technology

KB - Knowledge Base

MED - Mind Extension Deployment

ML -Machine Learning

Mgr - Manager

MLB - My Life Bits ( Bell, G 2005 )

MMI - Man-Machine Interface

NL - Natural Language

PA - Persona Agent

PM - Persona Model

POP - Principles Of Operations

PRTMM - Pattern Recognition Theory of Minds Machine

RDF-Resource Definition Language

SPA - Semantic Pointer Architecture (Eliasmith, C.. 2013)

QS- Quantified Self

UML - Unified Modeling Language

VLR-Visiting Location Register (Balston, D.M. 1993) 